\title{
Black hole cosmos and the micro cosmos
}

\author{
U. V.S. Seshavatharam*, S. Lakshminarayana \\ Honorary faculty, $i$-serve, alakapuri, hyderabad-35, AP, India \\ Dept. of nuclear physics, andhra university, visakhapatnam-03, AP, India \\ *Corresponding author E-mail: seshavatharam.uvs@gmail.com
}

\begin{abstract}
Point of 'big bang' can be considered as the center or characteristic reference point of cosmic expansion in all directions. If so, the existence of 'preferred direction' in the universe may not be wrong. Based on the Mach's principle, it can be suggested that, within the 'Hubble volume' overall distribution of 'Hubble mass' will explain the observed physical phenomena. With the discovered applications it is very clear to say that, without a joint and unified study of cosmology and atomic \& particle physics, one should not deny the concepts of black hole cosmology. The most interesting thing is that, at any given cosmic time, if the universe is a primordial growing black hole, then certainly its 'Schwarzschild radius' can be considered as its characteristic minimum size at that time. Clearly speaking, "forever rotating at light speed, high temperature and high angular velocity small sized primordial cosmic black hole gradually transforms into a low temperature and low angular velocity large sized massive primordial cosmic black hole". Independent of the redshift observations and considering the proposed relations, with a great confidence now one can start seeing/observing the universe as a primordial expanding and light speed rotating black hole. Based on the proposed relations and concepts of black hole cosmology, definitions of cosmic homogeneity and cosmic isotropy must be readdressed. It is also clear that, now the black hole universe is expanding in a decelerating mode at a very small rate in such a way that with current technology one cannot measure its deceleration rate. Finally it can be suggested that cosmic acceleration and dark energy can be considered as pure mathematical concepts and there exists no physical base behind their affirmation. For the most serious cosmologists this may be bitter news, but it is a fact. Authors hope that, by 2015 definitely this subject will come into main stream physics. With reference to Black hole cosmology, it can be suggested that, characteristic nuclear charge radius and the characteristic angular momentum of the revolving electron increase with cosmic time. In addition, characteristic nuclear charge radius is more fundamental than the reduced Planck's constant. The key point to be noted is that the Planck's constant can be considered as a cosmological constant.
\end{abstract}

Keywords: Mach's principle, hubble length, hubble volume, hubble mass, black hole cosmology, cmbr wavelength, nuclear radius, rms radius of proton, planck's constant, reduced planck's constant, fine structure ratio and avogadro number.

\section{Introduction}

It is very unfortunate to note that, rather than experimental confirmations [1], [2], the subject of modern cosmology is based on observations, mathematical calculations, hypothetical interpretations and less confident or ad-hoc conclusions [3]-[9]. To avoid this ambiguous situation, in this paper authors presented a unified approach that connects atom and the universe. Clearly speaking, by considering 'hydrogen atom' as a cosmological telescope - the current cosmological changes can be understood. In this attempt, many large numbers and many semi empirical relations will come into picture. Based upon one's individual scientific interest and imaginative power, each large number /semi empirical relation [10], [11] can be analyzed in different modes and with group discussions - finally a unified model of cosmology can be developed. In this regard, Chitkara University, Himachal Pradesh, India has recognized the previously proposed observations as a 'research paper' [12].

\section{Point of big bang may be the cosmic center}

As per the NASA web site information: "the Big Bang Model is a broadly accepted theory for the origin and evolution of our universe. It postulates those 12 to 14 billion years ago, the portion of the universe we can see today was only a 
few millimeters across. It has since expanded from this hot dense state into the vast and much cooler cosmos we currently inhabit. We can see remnants of this hot dense matter as the now very cold cosmic microwave background radiation which still pervades the universe and is visible to microwave detectors as a uniform glow across the entire sky. In addition the cosmic microwave background radiation, the remnant heat from the Big Bang, has a temperature which is highly uniform over the entire sky. This fact strongly supports the notion that the gas which emitted this radiation long ago was very uniformly distributed".

Extrapolation of the expansion of the Universe backwards in time using general relativity yields an infinite density and temperature at a finite time in the past [13]-[16].This singularity signals the breakdown of general relativity. How closely we can extrapolate towards the singularity is debated - certainly no closer than the end of the Planck echo. This singularity is sometimes called "the Big Bang", but the term can also refer to the early hot, dense phase itself which can be considered as the "birth" of our Universe. The earliest phases of the Big Bang are subject to much speculation. In the most common models the Universe was filled homogeneously and isotropic ally with an incredibly high energy density and huge temperatures and pressures and was very rapidly expanding and cooling.

In this regard the authors propose the following arguments. If expansion is taking place simultaneously in all directions at a uniform rate (at that time) about the point of big bang, then 'point of big bang' may be considered as the center or characteristic reference point of cosmic expansion in all directions. In this case, saying that there is no preferred direction in the expanding universe may not be correct. Due to the vastness of the universe, or due to the technological limits - right now one may not be in a position to see or feel the effects of the 'cosmic center' of expansion- but in future it may be possible. With reference to the increasing Hubble length if increasing Hubble volume is supposed to have center then it is not a big problem to think about the 'cosmic rotation'. In that case with reference to the current Hubble length, it is possible to say that, current Hubble volume rotates with constant light speed $c$ and angular velocity $H_{0}$. In this way the concept of 'repulsive gravity' can be replaced with cosmic constant light speed rotation [17].

Let $M_{0} \cong \frac{c^{3}}{2 G H_{0}}$ be a characteristic mass of a closed universe. Let its current volume density be $\left(\rho_{v}\right)_{0}$, current size be $R_{0}$, current matter density be $\left(\rho_{m}\right)_{0}$ and current thermal energy density be $a T_{0}^{4}$. By any reason, at present if $\left(\rho_{v}\right)_{0} \rightarrow \frac{a T_{0}^{4}}{c^{2}} \Rightarrow R_{0} \rightarrow 27.4 \frac{c}{H_{0}}$. Based on the elliptical or spiral galaxy's mass to light ratio if $\left(\rho_{v}\right)_{0} \rightarrow\left(\rho_{m}\right)_{0} \cong 8 \times 10^{-32}$ gram $/ \mathrm{cm}^{3} \Rightarrow R_{0} \rightarrow 5 \frac{c}{H_{0}}$ and if $\left(\rho_{v}\right)_{0} \rightarrow \frac{3 H_{0}^{2}}{8 \pi G} \Rightarrow R_{0} \rightarrow \frac{c}{H_{0}}$. In this way with reference to the current notion of $4.9 \%$ matter and $26.8 \%$ dark matter, the number 5 which is obtained by considering the matter density can be compared with $4.9 \%$ and the number 27.4 which is obtained by considering the thermal energy density can be compared with $26.8 \%$. In case of the number 5 , it is the matter density and hence there is no problem. In case of the number 27 , the very complicated thing to be understood is whether it is the 'dark matter' or the 'thermal energy density' that generates the number 27 to be confirmed. This reasoning will help in understanding both the closed and flat models of cosmology. If one is willing to think in this direction, to account for the third number $68.3 \%$, one must search for a physically observable new density that is just 15-16 times less than the current thermal energy density.

\section{Modified definitions of cosmic homogeneity and cosmic isotropy}

At any point within the Hubble volume, if a particular physical parameter's magnitude is measured to be same then it can be called as the cosmic 'homogeneity' for that physical parameter.

Within the Hubble volume, with reference to any two or more number of points, in any direction, if a particular physical parameter's magnitude is measured to be same then it can be called as the cosmic 'isotropy' for that physical parameter. It does not mean that, there is no 'preferred direction' in the universe.

\section{The cosmic 'critical density' and its dimensional analysis and the cosmic rotation}

Recent findings from the University of Michigan [18] suggest that the shape of the Big Bang might be more complicated than previously thought, and that the early universe spun on an axis. A left-handed and right-handed imprint on the sky as reportedly revealed by galaxy rotation would imply the universe was rotating from the very 
beginning and retained an overwhelmingly strong angular momentum. An anonymous referee who reviewed the paper for Physics Letters said, "In the paper the author claims that there is a preferred handedness of spiral galaxies indicating a preferred direction in the universe. Such a claim, if proven true, would have a profound impact on cosmology and would very likely result in a Nobel prize'.Galaxies spin, stars spin, and planets spin. So, why not the whole universe? The consequences of a spinning universe [18]-[32] seem to be profound, natural and 'cosmic collapse' can be prevented. Thus 'cosmic constant light speed rotation' [17], [33]-[41] can be considered as an alternative to the famous 'repulsive gravity' concept.

With a simple derivation it is possible to show that, Hubble's constant $H_{t}$ represents cosmological angular velocity. Authors presented this derivation in their published papers [12], [33], [34], [35], [36], [37]. Assume that, a planet of mass $\mathrm{M}$ and radius $\mathrm{R}$ rotates with angular velocity $\omega_{e}$ and linear velocity $v_{e}$ in such a way that, free or loosely bound particle of mass $\mathrm{m}$ lying on its equator gains a kinetic energy equal to potential energy as,

$$
\begin{aligned}
& \frac{1}{2} m v_{e}^{2}=\frac{G M m}{R} \\
& R \omega_{e}=v_{e}=\sqrt{\frac{2 G M}{R}} \text { and } \omega_{e}=\frac{v_{e}}{R}=\sqrt{\frac{2 G M}{R^{3}}}
\end{aligned}
$$

I.E. Linear velocity of planet's rotation is equal to free particle's escape velocity. Without any external power or energy, test particle gains escape velocity by virtue of planet's rotation. Using this idea, 'Black hole radiation' and 'origin of cosmic rays' can be understood. Note that if Earth completes one rotation in one hour then free particles lying on the equator will get escape velocity. Now writing $M=\frac{4 \pi}{3} R^{3} \rho_{e}$,

$$
\begin{aligned}
& \omega_{e}=\frac{v_{e}}{R}=\sqrt{\frac{8 \pi G \rho_{e}}{3}} \text { Or } \omega_{e}^{2}=\frac{8 \pi G \rho_{e}}{3} \\
& \text { Density, } \rho_{\mathrm{e}}=\frac{3 \omega_{\mathrm{e}}^{2}}{8 \pi \mathrm{G}}
\end{aligned}
$$

In real time, this obtained density may or may not be equal to the actual density. But the ratio, $\frac{8 \pi G \rho_{\text {real }}}{3 \omega_{\text {real }}^{2}}$ may have some physical significance. The most important point to be noted here, is that, as far as dimensions and units are considered, from equation (4), it is very clear that, proportionality constant being $\frac{3}{8 \pi G}$,

$$
\text { density } \propto(\text { angular velocity })^{2}
$$

Equation (4) is similar to "flat model concept" of cosmic "critical density"

$$
\rho_{c}=\frac{3 H_{t}^{2}}{8 \pi G}
$$

Comparing equations (4) and (6) dimensionally and conceptually, i.e.

$$
\begin{aligned}
& \rho_{e}=\frac{3 \omega_{e}^{2}}{8 \pi G} \text { with } \rho_{\mathrm{c}}=\frac{3 H_{\mathrm{t}}^{2}}{8 \pi \mathrm{G}} \\
& H_{t}^{2} \rightarrow \omega_{\mathrm{e}}^{2} \text { and } H_{t} \rightarrow \omega_{e}
\end{aligned}
$$

It is very clear that, dimensions of 'Hubble's constant' must be 'radian/second'. In any physical system under study, for any one 'simple physical parameter' there will not be two different units and there will not be two different physical meanings. This is a simple clue and brings "cosmic rotation" into picture. This is possible in a closed universe only. Cosmic models that depend on this "critical density" may consider 'angular velocity of the universe' in the place of 'Hubble's constant'. In the sense, with a great confidence 'cosmic rotation' can be included in the existing models of cosmology [17]-[32]. Then the term 'critical density' appears to be the 'volume density' of the closed and expanding universe.

\section{Applications of Hubble volume and Hubble mass in microscopic physics and the beginning of black hole cosmology}

Some cosmologists use the term 'Hubble volume' to refer to the volume of the observable universe. At any given time, the product of 'critical density' and 'Hubble volume' gives a characteristic cosmic mass and it can be called as the 
Hubble mass. Interesting thing is that, Schwarzschild radius of the 'Hubble mass' again matches with the Hubble length. Most of the cosmologists believe that this is merely a coincidence. If one is able to show the applications of 'Hubble mass' in different areas of fundamental physics, certainly it can be given more significance and top priority compared to the mysterious 'dark energy'. By increasing the number of applications of Hubble mass and Hubble volume [34] in other areas of fundamental physics, slowly and gradually and in progressive way concepts of Black hole Cosmology can be strengthened and can also be confirmed. Unknowingly the fundamental physical laws are being developed, being executed and being proven inside and under the background of a growing and light speed rotating black hole universe. If universe constitutes so many galaxies, if each galaxy constitutes a central fast growing and (light speed) spinning black hole and if black hole geometry is more intrinsic than its 'mass' and 'mass density', then considering universe as a 'growing and light speed rotating black hole' may not be far away from reality.

In 2013 February, using NASA's newly launched NuStar telescope and the European Space Agency's workhorse XMMNewton, an international team observed high-energy X-rays released by a super massive black hole in the middle of a nearby galaxy. They calculated its spin at close to the speed of light: 670 million mph [17]. This is really very good news for the beginning of 'Black hole cosmology. At any given cosmic time, 'Hubble length' can be considered as the gravitational or electromagnetic interaction range. Hubble volume and Hubble mass play a crucial role in quantum physics, nuclear physics, atomic physics and particle physics. The authors proposed various applications of Hubble mass in their previously published papers. By re-presenting the list of important discovered semi empirical relations, in this paper an attempt is made to fit and couple the CMBR wavelength and fine structure ratio.

\section{Strange things in modern cosmology}

Although Einstein published the details of his static, positively curved, matter filled model in the spring of 1917, he was dissatisfied with the model [16]. He believed that the cosmological constant was "gravely detrimental to the formal beauty of the theory". Hubble's 1929 paper on the redshift - distance relation [42] gave Einstein the necessary excuse for tossing "Lambda term" onto the rubbish heap. Since 1917, the cosmological constant has gone in and out of fashion, like sideburns or short skirts. It has been particularly fashionable during periods when the favoured value of the Hubble time has been embarrassingly short compared to the estimated ages of astronomical objects. Currently, the cosmological constant is very popular. It is intriguing to note that Friedmann published his first results, implying an expanding or contracting universe, seven years before Hubble published Hubble's Law in 1929. Unfortunately, Friedmann's papers received little notice at first. Even Einstein initially dismissed Friedmann's work as a mathematical curiosity, unrelated to the universe we actually live in. It wasn't until Hubble's results were published that Einstein acknowledged the reality of the expanding universe. Surprising thing is that in 1947 Hubble himself thought for a new mechanism for understanding the observed redshifts [43].It may be noted that, increased redshifts and increased distances forced Edwin Hubble to propose the Hubble's law. In fact there is no chance or scope or place for 'galaxy receding'. It is only our belief in its 'given' (Doppler shift based) interpretation. Even then, merely by estimating galaxy distance and without measuring galaxy receding speed, one cannot verify its acceleration. Clearly speaking: two mistakes are happening here. 1) Assumed galaxy receding speed is not being measured and not being confirmed. 2) Without measuring and confirming the galaxy receding speed, how can one say and confirm that it (galaxy) is accelerating. It is really speculative and unfortunate also. If 'Dark energy' is the major outcome of the 'accelerating universe', it is very important to note that - in understanding the basic concepts of unification or other fundamental areas of physics, role of dark energy is very insignificant. So far no ground based experiment confirmed the existence of dark energy. There is no single clue or definition or evidence to any of the natural physical properties of (the assumed) dark energy.

When it was proposed in 1948, at the beginning, no one believed in the existence of CMB radiation. The cosmic microwave background was first predicted in 1948 by Ralph Alpher and Robert Herman [44], [45], [46]. Alpher and Herman were able to estimate the temperature of the cosmic microwave background to be $50 \mathrm{~K}$, though two years later they re-estimated it as $280 \mathrm{~K}$. The 1948 results of Alpher and Herman were discussed in many physics settings through about 1955, when both left the Applied Physics Laboratory at Johns Hopkins University. The mainstream astronomical community, however, was not intrigued at the time by cosmology. Alpher and Herman's prediction was rediscovered by Yakov Zel'dovich in the early 1960s, and independently predicted by Robert Dicke [47] at the same time. The discovery of the Cosmic Microwave Background (CMB) by Arno Penzias and Robert Wilson in 1965 has entered cosmological folklore. Arno Penzias and Robert Wilson were surprised when they serendipitously discovered the Cosmic Microwave Background. At the time of their discovery, Penzias and Wilson were radio astronomers working at Bell Laboratories. The horn-reflector radio antenna which they used had previously been utilized to receive microwave signals of wavelength $_{s}=7.35 \mathrm{~cm}$, reflected from an orbiting communications satellite. Turning from telecommunications to astronomy, Penzias and Wilson found a slightly stronger signal than they expected when they turned the antenna toward the sky. They did everything they could think of to reduce 'noise' in their system. The stronger signal remained. It was isotropic and constant with time, so it couldn't be associated with an isolated celestial source. Wilson and Penzias were puzzled until they were put in touch with Robert Dicke and his research group at Princeton University. Dicke had 
deduced that the universe, if it started in a hot dense state, should now be filled with microwave radiation. Here the authors would like to stress the fact that, Penzias and Wilson were not aware of what they discovered. Drop in 'cosmic temperature' can be considered as a measure of cosmic expansion and 'rate of decrease in cosmic temperature' can be considered as a measure of cosmic 'rate of expansion'. But if rate of decrease in temperature is very small and is beyond the scope of current experimental verification, then the two possible states are: a) cosmic temperature is decreasing at a very slow rate and universe is expanding at a very slow rate and $b$ ) there is no 'observable' thermal expansion and there is no 'observable' cosmic expansion.

In theoretical physics, particularly in discussions of gravitation theories, Mach's principle [48], [49], [50] is the name given by Einstein to an interesting hypothesis often credited to the physicist and philosopher Ernst Mach. The idea is that the local motion of a rotating reference frame is determined by the large scale distribution of matter. There are a number of rival formulations of the principle. A very general statement of Mach's principle is 'local physical laws are determined by the large-scale structure of the universe'. This concept was a guiding factor in Einstein's development of the general theory of relativity. Einstein realized that the overall distribution of matter would determine the metric tensor, which tells the observer which frame is rotationally stationary. One of the main motivations behind formulating the general theory of relativity was to provide a mathematical description to the Mach's principle. However, soon after its formulation, it was realized that the theory does not follow Mach's principle. As the theoretical predictions were matching with the observations, Einstein believed that the theory was correct and did not make any further attempt to reformulate the theory to explain Mach's principle. Later on, several attempts were made by different researchers to formulate the theory of gravity based on Mach's principle. However most of these theories remain unsuccessful to explain different physical phenomena.

\section{To understand and re-interpret the Hubble's law}

It may be noted that, increased redshifts and increased distances forced Edwin Hubble to propose the Hubble's law. Since galaxy is not a point particle and if light is coming from the atoms of the gigantic galaxy, then cosmic redshift can be interpreted as an index of the galactic atomic 'light emission mechanism'. In no way it seems to be connected with 'galaxy receding'. If it is possible to show that, (from the observer) observed older galaxy's distance increases with its 'age', then the concepts 'galaxy receding' and 'accelerating universe' can be put for a revision at fundamental level. Whatever may be the expression, definitions of cosmic red shift seem to be ad-hoc and not absolute. With reference to our laboratory or our galaxy, the basic or original definition of present/current redshift seems to be:

$$
z_{0} \cong \frac{E_{0}-E_{G}}{E_{0}} \cong \frac{\lambda_{G}-\lambda_{0}}{\lambda_{G}} \cong\left(z_{x}\right)_{0} \leq 1 \text {. (say) }
$$

But not $z_{0} \cong \frac{E_{0}-E_{G}}{E_{G}} \cong \frac{\lambda_{G}-\lambda_{0}}{\lambda_{0}} \cong\left(z_{y}\right)_{0}$

With reference to the current definition of $z \cong\left(z_{y}\right)_{0}$, proposed $z \cong\left(z_{x}\right)_{0}$ can be expressed as follows.

$$
\left(z_{x}\right)_{0} \cong \frac{\left(z_{y}\right)_{0}}{1+\left(z_{y}\right)_{0}}
$$

Here $E_{0} \cong \frac{h c}{\lambda_{0}}$ is the energy of photon at our galaxy/laboratory and $E_{G} \cong \frac{h c}{\lambda_{G}}$ is the energy of photon at the observed galaxy when it was emitted. Similarly $\lambda_{G}$ is the wave length of light received from observed galaxy and $\lambda_{0}$ is the wave length of light in laboratory. Even though both relations are ad-hoc and not absolute definitions, compared to relation (10), relation (9) seems to be somewhat reliable. Very interesting thing is that, when redshift is very small (up to $z \approx 0.01$ ), both relations almost all will give the same result. Important point to be noticed is that, by Hubble's time the maximum redshift noticed was 0.003 and was less than 0.01 . One should not ignore this fact. Now the fundamental question to be answered is: which relation is correct: either relation (9) or relation (10)? Note that, present red shift $\left(z_{0}\right)$ will be directly proportional to age difference between our galaxy and observed galaxy or time taken by light to reach our galaxy from the observed galaxy $(\Delta t)$. Thus $z_{0} \propto \Delta t$ and

$$
z_{0} \cong H_{0} \Delta t \text {. }
$$

Here $H_{0}$ is the proportionality constant. In this way $H_{0}$ can be incorporated directly. Time taken by light to reach our galaxy or the age difference of our galaxy and observed galaxy can be expressed as, 


$$
\begin{aligned}
& \Delta t \cong \frac{z_{0}}{H_{0}} . \\
& c \Delta t \cong z_{0} \cdot \frac{c}{H_{0}}
\end{aligned}
$$

To confirm this, absolute methods (that are free from redshift) for estimating galaxy age can be considered. Then the basic and original definition of 'galaxy receding' and 'accelerating universe' concepts can be eliminated and a 'decelerating or expanded universe' concept can be continued without any difficulty. Hence with redshift concept - one may not be able to understand the actual rate of cosmic expansion and actual cosmic geometry [51], [52]

\section{Proposed Assumptions}

The possible assumptions in unified cosmic physics can be expressed in the following way. Please note that, with the proposed assumptions and observations/discoveries whether we are falling in an intellectual singularity or coming out from the intellectual singularity future may decide [40].

A) With reference to the elementary charge and with mass similar to the Planck mass, a new mass unit can be constructed in the following way. It can be called as the Coulomb mass.

$$
\left(M_{C}\right)^{ \pm} \cong \sqrt{\frac{e^{2}}{4 \pi \varepsilon_{0} G}} \cong 1.859210775 \times 10^{-9} \mathrm{Kg} \cong 1.042941 \times 10^{18} \mathrm{GeV} / \mathrm{c}^{2}
$$

It represents the characteristic mass of elementary charge in unification program. It can be considered as the seed of galactic matter or galactic central black hole. It can also be considered as the seed of any cosmic structures.

B) At any time Hubble length $\left(c / H_{t}\right)$ can be considered as the gravitational or electromagnetic interaction range.

C) At any time, $H_{t}$ being the angular velocity, universe can be considered as a growing and light speed rotating primordial black hole. Thus at any given cosmic time,

D)

$$
R_{t} \cong \frac{2 G M_{t}}{c^{2}} \cong \frac{c}{H_{t}} \quad \text { And } \quad M_{t} \cong \frac{c^{3}}{2 G H_{t}}
$$

When $M_{t} \rightarrow M_{C}, \quad R_{C} \cong \frac{2 G M_{C}}{c^{2}}$ and $H_{C} \cong \frac{c}{R_{C}} \cong \frac{c^{3}}{2 G M_{C}}$ can be considered as the characteristic initial physical measurements of the universe. Here the subscript $C$ refers to the initial conditions of the universe and can be called as the Coulomb scale. Similarly $R_{0} \cong \frac{2 G M_{0}}{c^{2}} \cong \frac{c}{H_{0}}$ and $\mathrm{M}_{0} \cong \frac{c^{3}}{2 G H_{0}}$ can be considered as the characteristic current physical measurements of the universe.

E) Reduced Planck's constant or the characteristic angular momentum of the revolving electron increases with cosmic time [53], [54] whereas the Planck's constant can be considered as a cosmological constant.

F) Characteristic nuclear size [55]-[59] increases with cosmic time. In this regard, to a great surprise, it is noticed that,

$$
\left(R_{0} R_{C}^{2}\right)^{\frac{1}{3}} \cong 9.97 \times 10^{-16} \mathrm{~m} \approx \text { One fermi }
$$

Independent of quantum mechanical constants, from this relation, $M_{0}$ and $H_{0}$ can be fitted directly.

Another interesting relation is

$$
R_{s} \cong \frac{G \sqrt{M_{0} \sqrt{m_{p} m_{e}}}}{c^{2}} \cong\left(\frac{m_{p}}{m_{e}}\right)^{\frac{1}{4}}\left(\frac{M_{0}}{m_{e}}\right)^{\frac{1}{2}} \frac{G m_{e}}{c^{2}} \cong(1.37 \text { to } 1.39) \times 10^{-15} \mathrm{~m}
$$

Where $m_{p}$ the rest is mass of proton and $m_{e}$ is the rest mass of electron.

\section{To understand the mystery of 'quantum' of angular momentum and 'rms' radius of proton}

To a great surprise it is noticed that 


$$
\frac{\hbar c}{G m_{p} \sqrt{M_{0} m_{e}}} \cong 0.99753 \cong 1
$$

Where $M_{0} \cong\left(c^{3} / 2 G H_{0}\right)$ and $H_{0}$ is close to $70 \mathrm{~km} / \mathrm{sec} / \mathrm{Mpc}$ [60]-[63]. This ratio is very close to unity! One should not ignore this strange and peculiar observation. From this relation it can be suggested that, along with the cosmic variable $H_{0}$, on the cosmological time scale, there exists one variable physical quantity in the presently believed atomic and nuclear physical constants. 'Rate of change' in its magnitude may be a measure of the present cosmic acceleration. Thus independent of the cosmic red shift and CMBR observations, from atomic and nuclear physics, cosmic acceleration can be verified. Above relation can be expressed as

$$
\hbar \cong \hbar_{0} \cong \sqrt{\frac{M_{0}}{m_{e}}} \cdot \frac{G m_{p} m_{e}}{c} \cong 1.0572 \times 10^{-34} \mathrm{~J} . \mathrm{sec}
$$

Here $\hbar \cong \hbar_{0}$ can be considered as the current magnitude of $\hbar_{t}$ and $\left(M_{0} / m_{e}\right)$ can be considered as the number of electrons in the present universe of mass, $M_{0} \cong\left(c^{3} / 2 G H_{0}\right)$.if so, present Hubble's constant can be expressed as

$$
H_{0} \cong \frac{G m_{p}^{2} m_{e} c}{2 \hbar_{0}^{2}} \cong 70.743 \mathrm{~km} / \mathrm{sec} / \mathrm{Mpc}
$$

It can be suggested that, 'quantum of angular momentum' may be due to the cosmological manifestation and 'discrete nature' of angular momentum may be due the discrete nuclear or atomic matter. In any bound system, 'operating force' only plays major role in maintaining the 'existence of the bound system' and 'angular momentum' is one of the result. If one is able to make the operating force as discrete, then automatically one can observe a discrete structure like discrete radii, discrete angular momentum and discrete energy levels. Alternatively if atomic nucleus constitutes any fixed number of protons and any fixed number of neutrons, it is possible to guess that- nuclear mass is discrete. If nuclear or atomic matter is discrete, it is also possible to have a discrete atomic structure. Another interesting relation is

$$
\hbar_{0}^{2} H_{0} \cong \hbar_{t}^{2} H_{t} \cong \frac{G m_{p}^{2} m_{e} c}{2} \cong \text { constant }
$$

Interesting thing is that,

$$
\frac{4 G M_{0}}{c^{2} R_{p}} \cong \sqrt{\frac{M_{0}}{m_{e}}}
$$

Where $R_{p}$ is the 'rms charge radius' of proton [58], [59]. This is another accurate relation that connects the universe and the atom and resembles the Einstein's famous space time curvature relation in case of bending of light ray. Thus $R_{p}$ can be expressed as

$$
R_{p} \cong \sqrt{\frac{m_{e}}{M_{0}}} \cdot \frac{4 G M_{0}}{c^{2}}
$$

It can be considered as the current ' $r m s$ ' radius of proton and can be expressed as

$$
\left(R_{p}\right)_{0} \cong \sqrt{\frac{m_{e}}{M_{0}}} \cdot \frac{4 G M_{0}}{c^{2}} \cong \frac{4 G \sqrt{M_{0} m_{e}}}{c^{2}}
$$

With different experimental methods (current) $R_{p}$ magnitude varies from $0.84184(67)$ fm to $0.895(18) \mathrm{fm}$. The two best quoted values of the rms radius of proton are $0.87680(690) \mathrm{fm}$ and $0.84184(67) \mathrm{fm}$. If so, present Hubble's constant can be expressed as

$$
H_{0} \cong \frac{2 G m_{p} m_{e}}{\left(R_{p}\right)_{0} \hbar_{0}} \cong \frac{4 \pi G m_{p} m_{e}}{\left(R_{p}\right)_{0} h} \cong(67.88 \text { to } 70.69) \mathrm{km} / \mathrm{sec} / \mathrm{Mpc}
$$

If $R_{p} \cong 0.84184(67) \mathrm{fm} \rightarrow \mathrm{H}_{0} \cong 70.69 \mathrm{~km} / \mathrm{sec} / \mathrm{Mpc}$ and if $R_{p} \cong 0.87680 \mathrm{fm} \rightarrow \mathrm{H}_{0} \cong 67.88 \mathrm{~km} / \mathrm{sec} / \mathrm{Mpc}$. from this relation it is very interesting to note that,

$$
\hbar_{0} \cong \frac{2 G m_{p} m_{e}}{\left(R_{p}\right)_{0} H_{0}} \cong\left(\frac{2 G m_{p}}{c^{2}\left(R_{p}\right)_{0}}\right) \frac{m_{e} c^{2}}{H_{0}} \cong \frac{h}{2 \pi}
$$


Here the expression $\left(\frac{2 G m_{p}}{c^{2}\left(R_{p}\right)_{0}}\right)$ represents the ratio of 'Black hole radius' of proton and the 'rms radius' of proton. If electron revolves round the proton of mass $m_{p}$ and rms radius $\left(R_{p}\right)$, this expression can be considered as a key tool in the combined study of atom and the universe. Now the famous Uncertainty relation can be expressed as

$$
\left.\frac{\hbar_{0}}{2}\right) \cong \frac{G m_{p} m_{e}}{\left(R_{p}\right)_{0} H_{0}} \cong\left(\frac{h}{4 \pi}\right.
$$

This equality may be an indication of the saturation of cosmic rate of expansion. Now this is the time to understand/resolve the following issues:

1) To classify $h, \hbar$ and $R_{p}$ into primary and secondary physical constants and to find their independent primordial existence with reference to each other.

2) Possibility of considering $\hbar$ and $R_{p}$ as cosmological variables.

3) Possibility of considering $h$ as a cosmological constant.

4) In the above relation to maintain the constancy of $\left(\frac{h}{4 \pi}\right)$ it is also possible to guess that,

$$
\left(R_{p}\right)_{0} H_{0} \cong\left(R_{p}\right)_{t} H_{t} \cong \frac{G m_{p} m_{e}}{(h / 4 \pi)} .
$$

Then at present,

$$
\left(R_{p}\right)_{0} \cong \frac{G m_{p} m_{e}}{(h / 4 \pi) H_{0}}
$$

\section{Direct fitting of CMBR energy density and the fine structure ratio}

In atomic and nuclear physics, the fine-structure ratio $(\alpha)$ is a fundamental physical constant [58], [64], [65], namely the coupling constant characterizing the strength of the electromagnetic interaction. Being a dimensionless quantity, it has a constant numerical value in all systems of units. If $\rho_{0} c^{2}$ is the present cosmic critical energy density and $a T_{0}^{4}$ is the present cosmic thermal energy density, it is noticed that,

$$
\ln \sqrt{\frac{a T_{0}^{4}}{\rho_{0} c^{2}} \cdot \frac{4 \pi \varepsilon_{0} G M_{0}^{2}}{e^{2}}} \cong\left(\frac{1}{\alpha}\right) \cong \ln \left[\left(1+\ln \left(\frac{M_{0}}{M_{C}}\right)\right)^{-1}\left(\frac{M_{0}}{M_{C}}\right)\right]
$$

At present, if $H_{0}$ is close to $71 \mathrm{~km} / \mathrm{sec} / \mathrm{Mpc}$ and $T_{0} \cong 2.725{ }^{\circ} \mathrm{K}$, obtained value of $(1 / \alpha)_{0}$ is 137.04773 . Note that, from unification point of view, till today role of dark energy or dark matter is unclear and undecided. Their laboratory or physical existence is also not yet confirmed. In this critical situation this application can be considered as a key tool in particle cosmology. Note that large dimensionless constants and compound physical constants reflect an intrinsic property of nature [10], [11]. Above relation takes the following form.

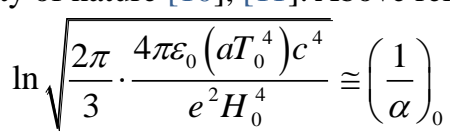

After simplification, it can be interpreted as follows. Total thermal energy in the present Hubble volume can be expressed as,

$$
\left(E_{T}\right)_{0} \cong a T_{0}^{4} \cdot \frac{4 \pi}{3}\left(\frac{c}{H_{0}}\right)^{3} \cong\left[\left(1+\ln \left(\frac{M_{0}}{M_{C}}\right)\right)^{-2} \frac{3 H_{0}^{2} c^{2}}{8 \pi G}\right] \cdot \frac{4 \pi}{3}\left(\frac{c}{H_{0}}\right)^{3}
$$

If $\left(c / H_{0}\right)$ is the present electromagnetic interaction range, then present electromagnetic potential can be expressed as

$$
\left(E_{e}\right)_{0} \cong \frac{e^{2}}{4 \pi \varepsilon_{0}\left(c / H_{0}\right)}
$$

Now inverse of the present fine structure ratio can be expressed as 


$$
\left(\frac{1}{\alpha}\right)_{0} \cong \ln \sqrt{\frac{\left(E_{T}\right)_{0}}{2\left(E_{e}\right)_{0}}}
$$

Here, in the RHS, denominator ' 2 ' may be a representation of total thermal energy in half of the cosmic sphere or thermal energy of any one pole of the cosmic sphere. This is a simple and direct application of the proposed assumptions. Thus at any cosmic time,

$$
\left(\frac{1}{\alpha}\right)_{t} \cong \ln \sqrt{\frac{\left(E_{T}\right)_{t}}{2\left(E_{e}\right)_{t}}}
$$

By any reason, at the initial conditions if thermal energy density equals to $\frac{3 H_{C}^{2} c^{2}}{8 \pi G},\left(\frac{1}{\alpha}\right)_{C} \rightarrow 0$. this is a very surprising and interesting result and needs a critical analysis.

\section{Equivalent cosmic matter density}

Approximate relation between cosmic volume density $\left(\rho_{v}\right)_{t}$ and matter density $\left(\rho_{m}\right)_{t}$ can be expressed as

$$
\left(\rho_{m}\right)_{t} \cong\left[1+\ln \sqrt{\frac{4 \pi \varepsilon_{0} G M_{t}^{2}}{e^{2}}}\right]^{-1}\left(\frac{3 H_{t}^{2}}{8 \pi G}\right) \cong\left[1+\ln \left(\frac{M_{t}}{M_{C}}\right)\right]^{-1}\left(\frac{3 H_{t}^{2}}{8 \pi G}\right)
$$

If $M_{t} \rightarrow M_{C} \cong \sqrt{\frac{e^{2}}{4 \pi \epsilon_{0} G}},\left(\rho_{m}\right)_{t} \rightarrow \frac{3 H_{C}^{2}}{8 \pi G}$. Instead of the 'Planck mass', initial conditions can be addressed with $M_{C} \cong \sqrt{\frac{e^{2}}{4 \pi \varepsilon_{0} G}}$. Note that, at present obtained matter density can be compared with the elliptical and spiral galaxy matter density. Based on the average mass-to-light ratio for any galaxy,

$$
\left(\rho_{m}\right)_{0} \cong 1.5 \times 10^{-32} \eta h_{0} \quad \mathrm{gram} / \mathrm{cm}^{3}
$$

where for any galaxy, $\langle\mathrm{M} / \mathrm{L}\rangle$ Galaxy $=\eta\langle\mathrm{M} / \mathrm{L}\rangle$ Sun and the number: $h_{0} \cong \frac{H_{0}}{100 \mathrm{Km} / \mathrm{sec} / \mathrm{Mpc}} \cong \frac{71.0}{100} \cong 0.71$. Note that elliptical galaxies probably comprise about $60 \%$ of the galaxies in the universe and spiral galaxies are thought to make up about $20 \%$ of the galaxies in the universe. Almost $80 \%$ of the galaxies are in the form of elliptical and spiral galaxies. For spiral galaxies, $\eta h_{0}^{-1} \cong 9 \pm 1$ and for elliptical galaxies $\eta h_{0}^{-1} \cong 10 \pm 2$. For our galaxy inner part $\eta h_{0}^{-1} \cong 6 \pm 2$. Thus the average $\eta h_{0}^{-1}$ is very close to 8 to 9 and its corresponding matter density is $(6.05$ to 6.8$) \times 10^{-32}$ $\operatorname{gram} / \mathrm{cm}^{3}$ [67], [68].

\section{Equivalent cosmic thermal energy density}

At any given cosmic time, ratio of cosmic volume energy density and cosmic thermal energy density can be expressed as

$$
\left(\frac{\rho_{v} c^{2}}{a T^{4}}\right)_{t} \cong\left[1+\ln \sqrt{\frac{4 \pi \varepsilon_{0} G M_{t}^{2}}{e^{2}}}\right]^{2} \cong\left[1+\ln \left(\frac{M_{t}}{M_{C}}\right)\right]^{2}
$$

This is an observation and can be considered as a discovery. If so, at any given cosmic time, equivalent thermal energy density can be expressed as

$$
a T_{t}^{4} \cong\left[1+\ln \left(\frac{M_{t}}{M_{C}}\right)\right]^{-2}\left(\frac{3 H_{t}^{2} c^{2}}{8 \pi G}\right)
$$

Please note that at present if present $H_{0}$ is close to $71.1 \mathrm{~km} / \mathrm{sec} / \mathrm{Mpc}$,

$$
a T_{0}^{4} \cong\left[1+\ln \left(\frac{M_{0}}{M_{C}}\right)\right]^{-2}\left(\frac{3 H_{0}^{2} c^{2}}{8 \pi G}\right) \cong 4.17283 \times 10^{-14} \mathrm{~J} / \mathrm{m}^{3}
$$


And corresponding current CMBR is temperature is $2.725^{\circ} \mathrm{K}$.

Here the fundamental question to be answered is- If $\hbar$ is a cosmological variable, how to confirm the constancy of the radiation constant $a$ ? If one is able to express the Wien's displacement constant $b$ in terms of electric charge $e$ and thermal energy constant $k_{B}$, then automatically $h c$ can be shown to be a cosmological constat related to electromagnetic and thermal energy and with this idea the Planck's quantum nature of energy can also be understood. From the above proposed relations it can be suggested that, $\hbar$ can be considered as a cosmological increasing atomic variable related to the revolving electron's angular momentum and $h$ can be considered as cosmological constant related to electromagnetic and thermal energy. For this purpose one can proceed in the following way. At any given cosmic time, it $a$ is the radiation energy constant and $b$ is the Wien's displacement constant, $a$ can be expressed as

$$
a \cong \frac{8 \pi^{5}}{15} \frac{k_{B}^{4}}{h^{3} c^{3}} \cong \frac{8 \pi^{5}}{15}\left(\frac{k_{B}^{3} b^{3}}{h^{3} c^{3}}\right) \frac{k_{B}}{b^{3}} \cong \frac{8 \pi^{5}}{15}\left(\frac{k_{B} b}{h c}\right)^{3} \frac{k_{B}}{b^{3}}
$$

It is noticed that, $\frac{8 \pi^{5}}{15}\left(\frac{k_{B}^{3} b^{3}}{h^{3} c^{3}}\right) \cong 1.3333995 \cong \frac{4}{3}$. Like photon's frequency-wavelength relation, $v \lambda=c$, in a classical approach, independent of the Planck's constant, at any given cosmic time, radiation constant $a$ can be expressed as

$$
a \cong \frac{4}{3} \cdot \frac{k_{B}}{b^{3}}
$$

This is a very sensitive point and can be understood from sections-13 and 14. Please note that Einstein used Wien's displacement law and Bohr's correspondence principle for deriving the Planck's law [58], [68]-[71]. Wien's law is based on classical theory and the correspondence principle assumes that the quantum theory and the classical theory coincide in centrum limits. From this it can be suggested that Wien's displacement law may be more fundamental than the Plank's law. With reference to the current magnitude of the Planck's constant, accurate value of the Wien's constant can be estimated and that obtained magnitude can be considered as a constant throughout the cosmic time. Further research and analysis may resolve the issue.

$$
\mathrm{F} M_{t} \rightarrow M_{C}, \text { thermal energy density } \cong a T_{C}^{4} \cong \frac{3 H_{C}^{2} c^{2}}{8 \pi G}
$$

It can be considered as the characteristic initial equivalent thermal energy density of the universe. At any given cosmic time, without considering the quantum theory of light, equivalent CMBR thermal energy density can be obtained in this way also. Really this is a miracle. If one is willing to think in this new direction, certainly other new relations can also be obtained. Its interpretation seems to be interesting. Compared to the complicated redshift observations, this proposal seems to be simple and reliable.

\section{Direct fitting of the wavelength of the CMB radiation}

Authors noticed two approximate methods for estimating the CMB radiation. Geometric mean of the two methods is fitting with the observational CMBR wavelength accurately [60]-[63]. Based on the wavelength and frequency relations of the Wien's displacement law [72] it is noticed that,

$$
\sqrt{\frac{c}{\lambda_{m} f_{m}}} \cong \sqrt{1.762} \cong 1.3274 \cong \frac{4}{3}
$$

Where $\lambda_{m}$ and $f_{m}$ are the wavelength and frequency corresponding to the maximum energy.

If $(\lambda)_{t}$ is the CMBR wavelength, at any given cosmic time

$$
\begin{aligned}
& (\lambda)_{t} \propto \sqrt{1+\ln \left(\frac{M_{t}}{M_{C}}\right)} \\
& (\lambda)_{t} \propto 2 \pi \sqrt{\left(\frac{2 G M_{t}}{c^{2}}\right) \cdot\left(\frac{2 G M_{C}}{c^{2}}\right)}
\end{aligned}
$$

Guessing in this way it is noticed that, if $\lambda_{f}$ is the wavelength corresponding to $\frac{d E_{v}}{d v}$ and $E_{v}$ is the total energy at all frequencies up to and including $v$, at any given cosmic time

$$
\left(\lambda_{f}\right)_{t} \cong \frac{4}{3} \cdot \sqrt{1+\ln \left(\frac{M_{t}}{M_{C}}\right)} \cdot \frac{4 \pi G \sqrt{M_{t} M_{C}}}{c^{2}}
$$


If $\lambda_{m}$ is the wavelength corresponding to $\frac{d E_{\lambda}}{d \lambda}$ and $E_{\lambda}$ is the total energy at all wavelengths up to and including $\lambda$, at any given cosmic time

$$
\left(\lambda_{m}\right)_{t} \cong \frac{3}{4} \cdot \sqrt{1+\ln \left(\frac{M_{t}}{M_{C}}\right)} \cdot \frac{4 \pi G \sqrt{M_{t} M_{C}}}{c^{2}}
$$

Thus it is possible to express both the wavelength relations in the following way.

$$
\left(\lambda_{f}, \lambda_{m}\right)_{t} \cong\left(\frac{4}{3}\right)^{ \pm 1} \cdot \sqrt{1+\ln \left(\frac{M_{t}}{M_{C}}\right)} \cdot \frac{4 \pi G \sqrt{M_{t} M_{C}}}{c^{2}}
$$

At present, if $H_{0}$ is close to $71 \mathrm{~km} / \mathrm{sec} / \mathrm{Mpc}$,

$$
\left(\lambda_{f}, \lambda_{m}\right)_{0} \cong\left(\frac{4}{3}\right)^{ \pm 1} \cdot \sqrt{1+\ln \left(\frac{M_{0}}{M_{C}}\right)} \cdot \frac{4 \pi G \sqrt{M_{0} M_{C}}}{c^{2}} \cong(1.90 \mathrm{~mm}, 1.069 \mathrm{~mm})
$$

These relations can be understood in the following semi empirical approach.

Method-1: With reference to the Wien's displacement law and if $\left(M_{C}\right)^{ \pm} \cong \sqrt{\frac{e^{2}}{4 \pi \varepsilon_{0} G}}$ represents a characteristic fundamental unified charged mass unit, wavelength of the most strongly emitted CMB radiation can be expressed as

$$
\left(\lambda_{m}\right)_{t} \cong\left[1+\ln \left(\frac{M_{t}}{M_{C}}\right)\right] \frac{G \sqrt{M_{t} M_{C}}}{c^{2}}
$$

Note that this expression is free from the 'radiation constants'. If $H_{0}$ is close to $70 \mathrm{~km} / \mathrm{sec} / \mathrm{Mpc}$, obtained (most strongly emitted) wavelength of the CMB radiation is $1.37 \mathrm{~mm}$.

Method-2: This method is based on the pair annihilation of $\left(M_{C}\right)^{ \pm}$. Pair particles creation and annihilation in 'free space' is an interesting idea. In the expanding universe, by considering the proposed charged $M_{C}$ and its pair annihilation as characteristic cosmic phenomena, origin of the isotropic CMB radiation can be addressed. Thermal energy can be expressed as

$$
k_{B} T_{t} \cong \sqrt{\frac{M_{C}}{M_{t}}} \cdot\left[\left(M_{C}\right)^{+}+\left(M_{C}\right)^{-}\right] c^{2} \cong \sqrt{\frac{M_{C}}{M_{t}}} \cdot 2 M_{C} c^{2}
$$

Based on the Wien's displacement constant,

$$
\left(\lambda_{m}\right)_{t} \cong \frac{b}{T_{t}} \cong \sqrt{\frac{M_{t}}{M_{C}}} \cdot \frac{b k_{B}}{2 M_{C} c^{2}}
$$

If $H_{0}$ is close to $70 \mathrm{~km} / \mathrm{sec} / \mathrm{Mpc}$, obtained (most strongly emitted) wavelength of the CMB radiation is $0.822 \mathrm{~mm}$.

Method-3: Considering the geometric mean wavelength of wavelengths obtained from methods-1 and 2, wave length of the most strongly emitted CMB radiation can be expressed as

$$
\begin{aligned}
& \left(\lambda_{m}^{2}\right)_{t} \cong\left[1+\ln \left(\frac{M_{t}}{M_{C}}\right)\right] \cdot\left(\frac{M_{t}}{M_{C}}\right) \cdot\left(\frac{b k_{B} G}{2 c^{4}}\right) \\
& \left(\lambda_{m}\right)_{t} \cong \sqrt{\left[1+\ln \left(\frac{M_{t}}{M_{C}}\right)\right] \cdot\left(\frac{M_{t}}{M_{C}}\right) \cdot\left(\frac{b k_{B} G}{2 c^{4}}\right)}
\end{aligned}
$$

At present, the measured CMBR wavelength can be expressed as

$$
\left(\lambda_{m}\right)_{0} \cong \sqrt{\left[1+\ln \left(\frac{M_{0}}{M_{C}}\right)\right] \cdot\left(\frac{M_{0}}{M_{C}}\right) \cdot\left(\frac{b k_{B} G}{2 c^{4}}\right)} \cong 1.064 \mathrm{~mm}
$$

Where $H_{0}$ is close to $70 \mathrm{~km} / \mathrm{sec} / \mathrm{Mpc}$. This is a very accurate fit and needs a special analysis. The most important point is that, as the black hole universe is expanding, its expansion rate can be checked with $\frac{d}{d t}\left(\lambda_{m}\right)_{t}$. Present observations indicate that, $\mathrm{CMB}$ radiation is smooth and uniform. Thus it can be suggested that, at present there is no detectable cosmic expansion or cosmic acceleration. Thus in a semi empirical approach, it can be suggested that, the wavelength of the CMB radiation follows the following three conditions. 


$$
\begin{aligned}
& \left(\lambda_{m}\right)_{t} \propto \sqrt{1+\ln \left(\frac{M_{t}}{M_{C}}\right)} \\
& \left(\lambda_{m}\right)_{t} \propto \sqrt{\frac{M_{t}}{M_{C}}} \\
& \left(\lambda_{m}\right)_{t} \propto \sqrt{\frac{b k_{B} G}{2 c^{4}}}
\end{aligned}
$$

$\sqrt{\frac{b k_{B} G}{2 c^{4}}} \cong 1.2855 \times 10^{-35} \mathrm{~m}$ seems to be a constant and can be considered as the characteristic classical thermal wave length. With reference to the assumed initial conditions, i.e. $M_{t} \rightarrow M_{C}$,

$$
\left(\lambda_{m}\right)_{C} \cong \sqrt{\frac{b k_{B} G}{2 c^{4}}} \cong 1.2855 \times 10^{-35} \mathrm{~m}
$$

At beginning, if $\left(\lambda_{m}\right)_{C} T_{C} \cong b$ and $a T_{C}^{4} \cong \frac{3 H_{C}^{2} c^{2}}{8 \pi G}$, it is also noticed that,

$$
\left(\lambda_{m}\right)_{C} \cong b\left(\frac{3 H_{C}^{2} c^{2}}{8 \pi G a}\right)^{-\frac{1}{4}} \cong 1.295 \times 10^{-35} \mathrm{~m}
$$

From this strange coincidence it can be suggested that,

$$
\sqrt{\frac{b k_{B} G}{2 c^{4}}} \cong b\left(\frac{8 \pi G a}{3 H_{C}^{2} c^{2}}\right)^{\frac{1}{4}}
$$

Where $a \cong \frac{4}{3} \cdot \frac{k_{B}}{b^{3}}, H_{C} \cong \frac{c^{3}}{2 G M_{C}}$ and $M_{C} \cong \sqrt{\frac{e^{2}}{4 \pi \varepsilon_{0} G}}$. It needs a very critical analysis. From this relation, $b$ can be expressed as

$$
b \cong \frac{512 \pi}{9} \frac{e^{2}}{4 \pi \varepsilon_{0} k_{B}} \cong 2.97385 \times 10^{-3}{ }^{0} \mathrm{~K} \cdot \mathrm{m}
$$

Even though here error is $3 \%$, based on the cosmological approach and based on the assumed initial conditions and present conditions of the universe it is possible to guess that, right from the beginning to the present time $b$ and hence $a$ both seem to be constants. Not only that, Planck's constant, Wien's displacement constant, Boltzmann's constant, speed of light and elementary charge can be expressed in a unified manner [74].

\section{To understand the Planck's quantum nature of energy and to consider $h c$ as a cosmological constant}

1) To understand the Planck's quantum nature of energy with $n=1,2,3$, mole interacting oscillators and

2) To consider $h c$ as a cosmological constant

Authors propose the following simple procedure. From the proposed idea if $a \cong \frac{4}{3} \frac{k_{B}}{b^{3}}$ and from Planck's quantum theory if $a \cong \frac{8 \pi^{5}}{15} \cdot \frac{k_{B}^{4}}{h^{3} c^{3}}, h c$ can be expressed as

$$
h c \cong\left(\frac{2 \pi^{5}}{5}\right)^{1 / 3} b k_{B} \cong 4.9652 b k_{B}
$$

Please note that from Planck's law of radiation [68]-[71], the number 4.9652 can be estimated with the expression

$$
x=\ln 5-\ln (5-x) \cong 4.96511423 \text {. }
$$

From relation (43) and considering the universal gas constant [58], [73], $b$ can be expressed as 


$$
b \cong \frac{512 \pi}{9} \cdot \frac{e^{2}}{4 \pi \varepsilon_{0} k_{B}} \cong \frac{512 \pi}{9} \cdot \frac{e^{2}}{4 \pi \varepsilon_{0}\left(R / N_{A}\right)}
$$

Where $R$ the universal is gas constant and $N_{A}$ is the Avogadro number [75]-[86] and can be considered as an index for one mole interacting oscillators. For $N_{A}$ oscillators i.e. for one mole number of oscillators

$$
b \cong \frac{512 \pi}{9} \cdot \frac{\left(N_{A}\right) e^{2}}{4 \pi \varepsilon_{0} R}
$$

It can be suggested that,

$$
\begin{aligned}
b & \propto N_{A} \\
b & \propto \frac{e^{2}}{4 \pi \varepsilon_{0} R}
\end{aligned}
$$

For $n=1,2,3, .$. mole interacting oscillators

$$
b \cong \frac{512 \pi}{9} \cdot \frac{\left(n \cdot N_{A}\right) e^{2}}{4 \pi \varepsilon_{0} R}
$$

Basically Wien's displacement constant seems to have a discrete nature. As the ratio $\left(N_{A} k_{B} / R\right) \cong 1$, now it can be suggested that, for one mole interacting oscillators

$$
\begin{aligned}
& h c \cong\left(\frac{2 \pi^{5}}{5}\right)^{\frac{1}{3}} \cdot b k_{B} \cong\left(\frac{2 \pi^{5}}{5}\right)^{\frac{1}{3}} \cdot \frac{512 \pi}{9} \cdot \frac{e^{2}}{4 \pi \varepsilon_{0}} \cong 887.39 \frac{e^{2}}{4 \pi \varepsilon_{0}} \\
& h \cong\left(\frac{2 \pi^{5}}{5}\right)^{\frac{1}{3}} \cdot \frac{b k_{B}}{c} \cong\left(\frac{2 \pi^{5}}{5}\right)^{\frac{1}{3}} \cdot \frac{512 \pi}{9} \cdot \frac{e^{2}}{4 \pi \varepsilon_{0} c}
\end{aligned}
$$

And as the ratio $\left(n N_{A} k_{B} / R\right) \cong n$, for $n=1,2,3, .$. mole interacting oscillators

$$
n(h c) \cong n \cdot\left(\frac{2 \pi^{5}}{5}\right)^{\frac{1}{3}} \cdot \frac{512 \pi}{9} \cdot \frac{e^{2}}{4 \pi \varepsilon_{0}} \cong\left(\frac{2 \pi^{5}}{5}\right)^{\frac{1}{3}} \cdot \frac{512 \pi}{9} \cdot \frac{n e^{2}}{4 \pi \varepsilon_{0}}
$$

Now the famous Planck's law for $n=1,2,3, .$. mole interacting oscillators can be expressed as

$$
n\left(\frac{h c}{\lambda}\right) \cong\left(\frac{2 \pi^{5}}{5}\right)^{\frac{1}{3}} \cdot \frac{512 \pi}{9} \cdot \frac{n e^{2}}{4 \pi \varepsilon_{0} \lambda} \cong 887.39 \frac{n e^{2}}{4 \pi \varepsilon_{0} \lambda}
$$

In this way the concept of 'discrete' quantum of energy can be understood. Not only that, 110 years of can a historical puzzle be expressed in terms of 'mole concept'. Authors are working in this direction also. Anyhow now this is time to revise the fundamental physical concepts of micro-macro cosmos.

\section{Hydrogen atom - revolving electron's total energy with the cosmological variable $h$}

The fundamental question to be answered is- is reduced Planck's an output of the atomic system or an input to the atomic system? From the above proposed observations in the following sections an attempt is made to address this problem. Here the very important issue is if $\mathrm{h}$ is assumed to be a cosmological variable, then one must explain both the variable nature of $\mathrm{h}$ and its quantum nature. In the earlier published papers [35]-[41], [75], [75]-[86] the authors proposed that, in atomic system, ratio of atomic gravitational constant $G_{A}$ and classical gravitational constant $G$ is close to the squared Avogadro number $N_{A}^{2}$.

$$
\frac{G_{A}}{G} \cong N_{A}^{2} \text { And } G_{A} \cong N_{A}^{2} G
$$

Note that, in any bound system, 'operating force' only plays a major role in maintaining the 'existence of the bound system' and 'angular momentum' is one of the results. If one is able to make the operating force as discrete, then automatically one can observe a discrete structure like discrete radii, discrete angular momentum and discrete energy levels. If $n=1,2,3$, based on the new idea $\left(n N_{A}\right)$, it is possible to introduce a characteristic force magnitude as follows. 


$$
\begin{aligned}
& F_{X} \cong \frac{c^{4}}{G_{A}} \\
& \left(F_{X}\right)_{n} \cong \frac{c^{4}}{\left(n N_{A}\right)^{2} G} \cong \frac{c^{4}}{n^{2} G_{A}}
\end{aligned}
$$

Where $n=1,2,3$, It plays a very interesting role in Super symmetric electroweak physics [78]-[85], [87]-[89], [90]. Another interesting observation can be expressed as follows.

$$
\begin{aligned}
& \left(R_{c}\right)_{0} \cong\left(\frac{\hbar c}{G_{A} m_{e}^{2}}\right)^{2}\left(\frac{2 G_{A} m_{e}}{c^{2}}\right) \\
& \left(\frac{\hbar c}{G_{A} m_{e}^{2}}\right)^{2} \cong\left(\frac{\hbar_{0} c}{G_{A} m_{e}^{2}}\right)^{2} \cong \frac{\left(R_{c}\right)_{0} c^{2}}{2 G_{A} m_{e}} \cong \frac{F_{X}\left(R_{c}\right)_{0}}{2 m_{e} c^{2}}
\end{aligned}
$$

With the proposal $G_{A} \cong N_{A}^{2} G$, from relations (20) and (79) it is noticed that,

$$
\begin{aligned}
& \left(R_{c}\right)_{0} \cong \frac{1}{N_{A}^{2}}\left(\frac{m_{p}}{m_{e}}\right)^{2}\left(\frac{c}{H_{0}}\right) \cong \frac{1}{N_{A}^{2}}\left(\frac{m_{p}}{m_{e}}\right)^{2}\left(\frac{2 G M_{0}}{c^{2}}\right) \\
& \frac{\left(R_{c}\right)_{0}}{2} \cong \frac{1}{N_{A}^{2}}\left(\frac{m_{p}}{m_{e}}\right)^{2}\left(\frac{c}{2 H_{0}}\right) \cong \frac{1}{N_{A}^{2}}\left(\frac{m_{p}}{m_{e}}\right)^{2}\left(\frac{G M_{0}}{c^{2}}\right)
\end{aligned}
$$

At present and at any given cosmic time it can be suggested that,

$$
\frac{c}{H_{0}\left(R_{c}\right)_{0}} \cong \frac{c}{H_{t}\left(R_{c}\right)_{t}} \cong N_{A}^{2}\left(\frac{m_{e}}{m_{p}}\right)^{2}
$$

With this ratio and $G_{A} \cong N_{A}^{2} G$ and $F_{X} \cong\left(c^{4} / G_{A}\right)$, electron, proton and neutron rest masses can be fitted. Please see section 19.

$$
\left(R_{c}\right)_{0} H_{0} \cong\left(R_{c}\right)_{t} H_{t}
$$

Please note that, along with modified SUSY concepts [78]-[85], [87]-[89], [90], the ratio $\frac{m_{e} c^{2}}{F_{X}\left(R_{c}\right)_{0}}$ plays a very interesting role in electroweak physics. At any given cosmic time, potential energy of electron in Hydrogen atom can be expressed as follows.

$$
\left(E_{P}\right)_{t} \cong-\left(\frac{\hbar_{t} c}{G_{A} m_{e}^{2}}\right)^{2} \frac{\sqrt{m_{p} m_{e}} \cdot c^{2}}{2 n^{2}} \cong-\frac{1}{4} \sqrt{\frac{m_{p}}{m_{e}}}\left(\frac{F_{X}}{n^{2}}\right)\left(R_{c}\right)_{t} \cong-\sqrt{\frac{m_{p}}{m_{e}}} \frac{F_{X}\left(R_{c}\right)_{t}}{4 n^{2}}
$$

Where $\hbar_{t} \cong \frac{1}{\alpha_{t}} \cdot \frac{e^{2}}{4 \pi \varepsilon_{0} c}$ Or $\hbar_{t} \cong \sqrt{\frac{M_{t}}{m_{e}}} \cdot \frac{G m_{p} m_{e}}{c}$ to be confirmed by a suitable model and $2 n^{2}$ represents the total number of possible electrons in any principal quantum number $n=1,2,3, \ldots$ for further information please see the following sections $-16,17$ and 18. By any reason at any given cosmic time if revolving electron's kinetic energy is numerically equal to half the potential energy, then revolving electron's total energy can be expressed as follows.

$$
\left(E_{T}\right)_{t} \cong-\left(\frac{\hbar_{t} c}{G_{A} m_{e}^{2}}\right)^{2} \frac{\sqrt{m_{p} m_{e}} \cdot c^{2}}{4 n^{2}} \cong-\sqrt{\frac{m_{p}}{m_{e}}} \frac{F_{X}\left(R_{c}\right)_{t}}{8 n^{2}}
$$

With usual notation, from Bohr's theory of hydrogen atom, based on the jumping nature of electron, at any cosmic time emitted photon energy can be expressed as follows.

$$
\begin{aligned}
& \left(E_{\text {photon }}\right)_{t} \cong\left(\frac{\hbar_{t} c}{G_{A} m_{e}^{2}}\right)^{2} \frac{\sqrt{m_{p} m_{e}} \cdot c^{2}}{4}\left(\frac{1}{n_{1}^{2}}-\frac{1}{n_{2}^{2}}\right) \\
& \cong\left(\frac{\hbar_{t}}{\hbar_{0}}\right)^{2}\left\{\left(\frac{\hbar_{0} c}{G_{A} m_{e}^{2}}\right)^{2} \frac{\sqrt{m_{p} m_{e}} \cdot c^{2}}{4}\left(\frac{1}{n_{1}^{2}}-\frac{1}{n_{2}^{2}}\right)\right\} \\
& \cong\left(\frac{\hbar_{t}}{\hbar_{0}}\right)^{2}\left(E_{\text {photon }}\right)_{0}
\end{aligned}
$$

Where $n_{2}>n_{1}$. In this way in a cosmological approach it can be suggested that, 
1) The definition $\hbar \cong \frac{h}{2 \pi}$ seems to be an accidental coincidence. This equality i.e. $\left(\hbar_{0} \rightarrow \frac{h}{2 \pi}\right)$ may be an indication of the saturation of cosmic rate of expansion also.

2) At beginning $\hbar_{t} \rightarrow 0$ and as cosmic time increases $\hbar_{t}$ magnitude increases. $d \hbar / d t$ Or $d(1 / \alpha) / d t$ can be considered as a measure of cosmic rate of expansion.

3) During cosmic evolution, as cosmic time increases, hydrogen atom emits photons with increased quanta of energy. Thus past light quanta emitted from old galaxy will have less energy and show a red shift with reference to our galaxy.

4) During journey light quanta will not lose energy and there will be no change in light wavelength.

5) At any given cosmic time, for any galaxy cosmic redshift can be expressed as

$z_{0} \cong \frac{\left(E_{\text {photon }}\right)_{0}-\left(E_{\text {photon }}\right)_{t}}{\left(E_{\text {photon }}\right)_{0}} \cong \frac{\left(h c / \lambda_{0}\right)-\left(h c / \lambda_{G}\right)}{\left(h c / \lambda_{0}\right)} \cong 1-\left(\frac{\lambda_{0}}{\lambda_{G}}\right) \leq 1$

Where $\lambda_{0}$ is the light wavelength at our laboratory and $\lambda_{G}$ is the observed wavelength of the remote light. From atomic physics point of view from relation (88) the same redshift can be expressed in the following way.

$$
z_{0} \cong \frac{\left(E_{\text {photon }}\right)_{0}-\left(E_{\text {photon }}\right)_{t}}{\left(E_{\text {photon }}\right)_{0}} \cong 1-\left(\frac{\hbar_{t}}{\hbar_{0}}\right)^{2} \cong 1-\left(\frac{H_{0}}{H_{t}}\right) \leq 1
$$

Equating above relations (88) and (89)

$$
1-\left(\frac{\lambda_{0}}{\lambda_{G}}\right) \cong 1-\left(\frac{\hbar_{t}}{\hbar_{0}}\right)^{2} \cong 1-\left(\frac{H_{0}}{H_{t}}\right) \rightarrow \hbar_{t} \cong \sqrt{\frac{\lambda_{0}}{\lambda_{G}}} \cdot \hbar_{0} \text { and } H_{t} \cong\left(\frac{\lambda_{G}}{\lambda_{0}}\right) \cdot H_{0}
$$

\section{Discrete force, discrete radii and discrete energy levels in Hydrogen atom}

The basic ideas can be stated as follows.

1) Nuclear charge radius increases with cosmic time.

2) Nuclear charge radius is more fundamental than the presently believed 'reduced Planck's constant'.

3) At any given cosmic time, Reduced Planck's constant is a function of increasing nuclear charge radius.

4) Within the hydrogen atom, nuclear charge radius, proton mass and electron mass play a vital role.

5) If nuclear mass and nuclear charge radius both are assumed to play a fundamental role in the formation of atom and cause electron to revolve round the nucleus, then reduced Planck's constant can be assumed as cosmological compound atomic physical variable.

Proposed discrete force $\left(F_{X}\right)_{n} \cong \frac{c^{4}}{\left(n N_{A}\right)^{2} G} \cong \frac{c^{4}}{n^{2} G_{A}}$ plays a vital role in the observed discrete energy spectrum of Hydrogen atom. It is the root cause of the observed discrete angular momentum of electron.

In a cosmological approach, at present cosmic time, the discrete Bohr radii can be expressed as follows.

$$
\begin{aligned}
& \left(a_{n}\right)_{0} \propto \frac{e^{2}}{4 \pi \varepsilon_{0}\left(R_{p}\right)_{0}} \\
& \left(a_{n}\right)_{0} \propto \sqrt{\frac{m_{e}}{m_{p}}} \\
& \left(a_{n}\right)_{0} \propto\left(\frac{2 R_{c}}{R_{p}}\right)_{0} \\
& \left(a_{n}\right)_{0} \propto \frac{n^{2} G_{A}}{c^{4}}
\end{aligned}
$$

Thus, $\left(a_{n}\right)_{0} \cong \sqrt{\frac{m_{e}}{m_{p}}}\left(\frac{2 R_{c}}{R_{p}}\right)_{0} \frac{e^{2}}{4 \pi \varepsilon_{0}\left(R_{p}\right)_{0}}\left(\frac{n^{2} G_{A}}{c^{4}}\right)$

Numerically by considering the experimental values of the unit nuclear charge radius and the 'rms' radius of proton it is noticed that, 


$$
\left(\frac{R_{c}}{R_{p}}\right)_{0} \cong \frac{(1.21 \text { to } 1.23) \mathrm{fm}}{(0.84 \text { to } 0.87) \mathrm{fm}} \cong \sqrt{2}
$$

If $\left(R_{p} H_{0}\right)$ and $\left(R_{c} H_{0}\right)$ both are cosmological constants, at any time the ratio $\left(\frac{R_{c}}{R_{p}}\right)_{t}$ can also be considered as a cosmological constant. Hence, it can be suggested that, at any cosmic time,

$$
\left(R_{p}\right)_{t} \cong\left(\frac{R_{c}}{\sqrt{2}}\right)_{t} \text { and }\left(R_{p}\right)_{0} \cong\left(\frac{R_{c}}{\sqrt{2}}\right)_{0}
$$

Now the discrete Bohr radii can be expressed as follows.

$$
\left(a_{n}\right)_{0} \cong 4 \sqrt{\frac{m_{e}}{m_{p}}} \frac{e^{2}}{4 \pi \varepsilon_{0}\left(R_{c}\right)_{0}}\left(\frac{n^{2} G_{A}}{c^{4}}\right)
$$

Potential energy of the revolving electron can be simplified into the following very simple form.

$$
\left(E_{P}\right)_{0} \cong-\frac{e^{2}}{4 \pi \varepsilon_{0}\left(a_{n}\right)_{0}} \cong-\sqrt{\frac{m_{p}}{m_{e}}} \frac{\left(R_{c}\right)_{0} c^{4}}{4 n^{2} G_{A}}
$$

Based on the relation, at present,

$$
\frac{e^{2}}{4 \pi \varepsilon_{0}\left(a_{n}\right)_{0}^{2}} \cong \frac{m_{e}\left(v_{n}^{2}\right)_{0}}{\left(a_{n}\right)_{0}}
$$

Revolving electron's kinetic energy can be expressed as follows.

$$
\left(E_{K}\right)_{0} \cong \frac{e^{2}}{8 \pi \varepsilon_{0}\left(a_{n}\right)_{0}} \cong \sqrt{\frac{m_{p}}{m_{e}}} \frac{\left(R_{c}\right)_{0} c^{4}}{8 n^{2} G_{A}}
$$

Hence total energy of the revolving electron can be expressed as follows.

$$
\left(E_{T}\right)_{0} \cong\left(E_{P}\right)_{0}+\left(E_{K}\right)_{0} \cong-\sqrt{\frac{m_{p}}{m_{e}}} \frac{\left(R_{c}\right)_{0} c^{4}}{8 n^{2} G_{A}}
$$

Considering the jumping nature of electron, at present emitted photon energy can be expressed as follows.

$$
\left(E_{\text {photon }}\right)_{0} \cong \sqrt{\frac{m_{p}}{m_{e}}} \frac{\left(R_{c}\right)_{0} c^{4}}{8 G_{A}}\left(\frac{1}{n_{1}^{2}}-\frac{1}{n_{2}^{2}}\right) \cong \frac{h c}{\lambda_{0}}
$$

Wavelength of the emitted photon in hydrogen atom can be expressed as follows.

$$
\lambda_{0} \cong\left(\frac{m_{e}}{m_{p}}\right)^{\frac{1}{2}}\left(\frac{8 G_{A} h}{c^{3}\left(R_{c}\right)_{0}}\right)\left(\frac{1}{n_{1}^{2}}-\frac{1}{n_{2}^{2}}\right)^{-1}
$$

Corresponding revolving speeds of electron can be expressed as follows.

$$
\left(v_{n}\right)_{0} \cong\left(\frac{m_{p}}{m_{e}}\right)^{\frac{1}{4}} \sqrt{\frac{\left(R_{c}\right)_{0} c^{4}}{4 n^{2} G_{A} m_{e}}} \cong \sqrt{\frac{1}{2 n^{2}}}\left(\frac{m_{p}}{m_{e}}\right)^{\frac{1}{4}} \sqrt{\frac{\left(R_{c}\right)_{0} c^{2}}{2 G_{A} m_{e}}} \cdot c \cong \frac{1}{n}\left(\frac{m_{p}}{m_{e}}\right)^{\frac{1}{4}} \sqrt{\frac{\left(R_{c}\right)_{0} c^{2}}{4 G_{A} m_{e}}} \cdot c
$$

Corresponding discrete angular momentum of electron can be expressed as follows [91], [92].

$$
\begin{aligned}
& m_{e}\left(v_{n}\right)_{0}\left(a_{n}\right)_{0} \cong\left(\frac{m_{e}}{m_{p}}\right)^{\frac{1}{4}} \sqrt{\frac{4 n^{2} G_{A} m_{e}}{\left(R_{c}\right)_{0} c^{4}}} \frac{e^{2}}{4 \pi \varepsilon_{0}} \cong\left(\frac{m_{e}}{m_{p}}\right)^{\frac{1}{4}} \sqrt{\frac{4 n^{2} G_{A} m_{e} c^{2}}{\left(R_{c}\right)_{0} c^{4}}}\left(\frac{e^{2}}{4 \pi \varepsilon_{0} c}\right) \\
& m_{e}\left(v_{n}\right)_{0}\left(a_{n}\right)_{0} \cong \sqrt{2 n^{2}}\left(\frac{m_{e}}{m_{p}}\right)^{\frac{1}{4}} \sqrt{\frac{2 G_{A} m_{e}}{\left(R_{c}\right)_{0} c^{2}}}\left(\frac{e^{2}}{4 \pi \varepsilon_{0} c}\right) \cong n\left[\left(\frac{m_{e}}{m_{p}}\right)^{\frac{1}{4}} \sqrt{\frac{4 G_{A} m_{e}}{\left(R_{c}\right)_{0} c^{2}}}\left(\frac{e^{2}}{4 \pi \varepsilon_{0} c}\right)\right] \cong n \hbar_{0}
\end{aligned}
$$

From these relations it can be suggested that,

1) $\frac{2 G_{A} m_{e}}{\left(R_{c}\right)_{0} c^{2}}$ and $\frac{4 G_{A} m_{e}}{\left(R_{c}\right)_{0} c^{2}}$ Play a crucial role in atomic space-time curvature [93], [94].

2) Atomic space-time curvature is $N_{A}^{2}$ times the Schwarzschild space- time curvature.

3) Quantum interaction strength is $N_{A}^{2}$ times the gravitational interaction 
4) With reference to the ratio $\frac{2 G_{A} m_{e}}{\left(R_{c}\right)_{0} c^{2}}, 2 n^{2}$ can be identified as a peculiar term in atomic system.

5) $\left(\hbar_{0}\right)$ can be considered as the output of the atomic system but not the input.

Now inverse of fine structure ratio can be expressed as follows.

$$
\left(\frac{1}{\alpha_{n}}\right)_{0} \cong \sqrt{2 n^{2}}\left(\frac{m_{e}}{m_{p}}\right)^{\frac{1}{4}} \sqrt{\frac{2 G_{A} m_{e}}{\left(R_{c}\right)_{0} c^{2}}}
$$

At the ground state, with reference to the present cosmological unit nuclear charge radius $\left(R_{c}\right)_{0}$,

$$
\left(\frac{1}{\alpha}\right)_{0} \cong\left(\frac{m_{e}}{m_{p}}\right)^{\frac{1}{4}} \sqrt{\frac{4 G_{A} m_{e}}{\left(R_{c}\right)_{0} c^{2}}}
$$

\section{To understand the Planck's discrete quantum hypothesis}

From above relations (29), (81) and (96) $h c$ can be expressed with $2.14 \%$ error in the following form.

$$
h c \cong 4 \pi\left(\frac{R_{c}}{R_{p}}\right)_{0}\left(\frac{m_{e}}{m_{p}}\right) \frac{\left(m_{e} c^{2}\right)^{2}}{F_{X}} \cong \sqrt{2} \cdot 4 \pi\left(\frac{m_{e}}{m_{p}}\right) G_{A} m_{e}^{2}
$$

It can be understood as follows.

$$
\begin{aligned}
& h c \propto\left(\frac{m_{e}}{m_{p}}\right) \\
& h c \propto m_{e} c^{2} \\
& h c \propto 2 \pi\left(\frac{2 G_{A} m_{e}}{c^{2}}\right) \cong \frac{4 \pi G_{A} m_{e}}{c^{2}} \\
& h \propto\left(\frac{R_{c}}{R_{p}}\right)_{0} \cong \sqrt{2} \\
& h \cong \sqrt{2}\left(\frac{4 \pi G_{A} m_{e}^{3}}{m_{p} c}\right) \\
& \frac{h}{4 \pi} \cong \frac{\sqrt{2} G_{A} m_{e}^{3}}{m_{p} c}
\end{aligned}
$$

Now by considering the arrangement of electrons within the atomic principal quantum shells, the original discrete quantum hypothesis can be understood in the following way. Let us guess that, for a black body of any atomic number, let the minimum number of participating electrons be 'one' and the maximum number of participating electrons be $2 n^{2}$. Then the geometric mean number of participating electrons can be represented by $\sqrt{2 n^{2}}$. In the above relations if one is willing to replace $\sqrt{2}$ by $\sqrt{2 n^{2}}$, automatically the Planck's discrete quantum hypothesis [68], [90], [91] can be understood and can be represented as follows.

$$
n(h c) \cong \sqrt{2 n^{2}} \frac{4 \pi G_{A} m_{e}^{3}}{m_{p}}
$$

Based on this logic, with 1\% error Avogadro number can be expressed as follows.

$$
N_{A} \cong\left(\frac{1}{2}\right)^{\frac{1}{4}} \sqrt{\frac{h c m_{p}}{4 \pi G m_{e}^{3}}} \cong 6.0875 \times 10^{23}
$$

From relations (103) and (110), wavelength of the emitted photon in hydrogen atom can be expressed as follows.

$$
\lambda_{0} \cong 8 \pi \sqrt{2}\left(\frac{m_{e}}{m_{p}}\right)^{\frac{3}{2}}\left(\frac{4 G_{A}^{2} m_{e}^{2}}{\left(R_{c}\right)_{0} c^{4}}\right)\left(\frac{1}{n_{1}^{2}}-\frac{1}{n_{2}^{2}}\right)^{-1} \cong 8 \pi\left(\frac{m_{e}}{m_{p}}\right)^{\frac{3}{2}}\left(\frac{4 G_{A}^{2} m_{e}^{2}}{\left(R_{p}\right)_{0} c^{4}}\right)\left(\frac{1}{n_{1}^{2}}-\frac{1}{n_{2}^{2}}\right)^{-1}
$$


With reference to the relation (115), independent of cosmological changes, Based on the Bohr's theory of Hydrogen atom, with $4.5 \%$ error Bohr radii can be expressed as follows.

$$
a_{n} \cong 2 n^{2}\left(\frac{m_{e}}{m_{p}}\right)^{2}\left(\frac{2 G_{A} m_{e}}{c^{2}}\right)^{2}\left(\frac{4 \pi \varepsilon_{0} m_{e} c^{2}}{e^{2}}\right)
$$

This relation is very simple to understand. Here $\left(\frac{2 G_{A} m_{e}}{c^{2}}\right)$ represents the black hole radius of electron where the operating gravitational constant is $G_{A} \cong N_{A}^{2} G$ and $\left(\frac{e^{2}}{4 \pi \varepsilon_{0} m_{e} c^{2}}\right)$ represents the classical radius of electron. $2 n^{2}$ Represents the maximum number of possible electrons in any principal quantum number. Potential energy of electron can be expressed as follows.

$$
\left(E_{P}\right)_{n} \cong \frac{1}{2 n^{2}}\left(\frac{m_{p}}{m_{e}}\right)^{2}\left(\frac{c^{2}}{2 G_{A} m_{e}}\right)^{2}\left(\frac{e^{2}}{4 \pi \varepsilon_{0} m_{e} c^{2}}\right)\left(\frac{e^{2}}{4 \pi \varepsilon_{0}}\right)
$$

Aim of proposing these relations here is to show that, $a_{n} \propto 2 n^{2}, \quad\left(E_{P}\right) \propto \frac{1}{2 n^{2}}$ and $a_{n} \propto N_{A}^{4}, \quad\left(E_{P}\right) \propto \frac{1}{N_{A}^{4}}$. From relations (98) and (99) it is possible to show that, in a cosmological approach $\left(a_{n}\right)_{0} \propto 2 n^{2}, \quad\left(E_{P}\right)_{0} \propto \frac{1}{2 n^{2}}$ and $\left(a_{n}\right)_{0} \propto N_{A}^{2}, \quad\left(E_{P}\right)_{0} \propto \frac{1}{N_{A}^{2}}$. From this it can be suggested that, with the proposed two concepts $\left(\frac{c^{4}}{n^{2} G_{A}}\right)$ and $\frac{2 G_{A} m_{e}}{\left(R_{c}\right)_{0} c^{2}}$, mystery of quantum theory of light and discrete energy levels of electron in hydrogen atom both can be studied in a unified manner. Finally with group discussions in national and international science conferences, the odd concepts of modern quantum physics can be eliminated.

\section{To fit the Hubble's constant, Avogadro number, gravitational constant and the proton $\&$ electron rest masses}

Semi empirically it is noticed that at any given cosmic time,

$$
\frac{m_{p}}{m_{e}} \cong N_{A} \sqrt{\frac{\left(R_{c}\right)_{0} H_{0}}{c}} \cong N_{A} \sqrt{\frac{\left(R_{c}\right)_{t} H_{t}}{c}}
$$

Where $\left(R_{c}\right)_{0}$ and $\left(R_{c}\right)_{t}$ represent the current and past nuclear charge radii and $H_{0}$ and $H_{t}$ represent the current and past Hubble constants. This is a direct and simple relation. Semi empirically in a trial-error approach it is also noticed that

$$
\ln \sqrt{\frac{e^{2}}{4 \pi \varepsilon_{0} G m_{p}^{2}}} \cong \ln \left(\frac{M_{C}}{m_{p}}\right) \cong \sqrt{\frac{m_{p}}{m_{e}}-\ln \left(N^{2}\right)}
$$

Where $m_{p}$ is the proton rest mass and $m_{e}$ is the electron rest mass. Considering this as a characteristic relation, proton rest mass can be fitted accurately in the following way.

$$
\sqrt{\frac{e^{2}}{4 \pi \varepsilon_{0} G m_{p}^{2}}} \cong \frac{M_{C}}{m_{p}} \cong e^{\sqrt{\frac{m_{p}}{m_{e}}-\ln \left(N^{2}\right)}}
$$

The gravitational constant can be expressed as follows.

$$
G \cong\left(e^{\sqrt{\frac{m_{p}}{m_{e}}-\ln \left(N^{2}\right)}}\right)^{-2} \cdot \frac{e^{2}}{4 \pi \varepsilon_{0} m_{p}^{2}}
$$

Avogadro number can be expressed as follows.

$$
N \cong \sqrt{\exp \left[\frac{m_{p}}{m_{e}}-\left(\ln \sqrt{\frac{e^{2}}{4 \pi \varepsilon_{0} G m_{p}^{2}}}\right)^{2}\right]} \cong \sqrt{\exp \left[\frac{m_{p}}{m_{e}}-\left(\ln \left(\frac{M_{C}}{m_{p}}\right)\right)^{2}\right]}
$$


Independent of any system of units, the characteristic relation that connects the gram mole and the unified atomic mass unit can be expressed in the following way.

$$
G_{A} m_{u}^{2} \cong G M_{x}^{2} \text { And } M_{x} \cong \sqrt{\frac{G_{A}}{G}} \cdot m_{u} \cong N_{A} \cdot m_{u}
$$

Here $m_{u} \cong 1.66054 \times 10^{-27} \mathrm{~kg}$ is the unified atomic mass unit [84], $M_{x} \cong 0.001 \mathrm{~kg}$ is the gram mole expressed in $\mathrm{kg}$.

\section{To fit the rest masses of electron, proton and neutron}

$$
\text { Let, } \begin{aligned}
x & \cong \ln \sqrt{\frac{c}{\left(R_{c}\right)_{0} H_{0}}} \cong \ln \sqrt{\frac{c}{\left(R_{c}\right)_{t} H_{t}}} \\
y & \cong x\left[1-\frac{1}{\ln (x \sqrt{y})}\right]
\end{aligned}
$$

With trial and error, if $x$ is known, value of $y$ can be fitted. Let $\left(R_{c}\right)_{0} \cong 1.22 \mathrm{fm}$ and $H_{0} \cong 71 \mathrm{~km} / \mathrm{sec} / \mathrm{Mpc}$. Then $x \cong 47.2359$. please see the following table 1 for fitting the value of $y$.

Table 1: To fit the value of $y$.

\begin{tabular}{ccc}
\hline Trial No & Assumed value of $y$. & Obtained value of $y$. \\
\hline 1 & 47.2359 & 39.06746 \\
2 & 39.06746 & 38.931124 \\
3 & 38.931124 & 38.9285707 \\
4 & 38.9285707 & 38.9285228 \\
5 & 38.9285228 & 38.9285219 \\
6 & 38.9285219 & 38.92852184 \\
7 & 38.92852184 & 38.92852184 \\
\hline
\end{tabular}

At $7^{\text {th }}$ trial, assumed value of $y$ is equal to the obtained value of $y=38.92852184$. With these numerical values of $x$ and $y$, to a very good accuracy it is noticed that,

$$
\begin{aligned}
& \frac{e^{2}}{4 \pi \varepsilon_{0} G m_{p} m_{e}} \cong \frac{1}{x}\left(\frac{c}{\left(R_{c}\right)_{0} H_{0}}\right) \cong \frac{1}{x}\left(\frac{c}{\left(R_{c}\right)_{t} H_{t}}\right) \\
& \frac{e^{2}}{4 \pi \varepsilon_{0} G m_{e}^{2}} \cong y\left(\frac{c}{\left(R_{c}\right)_{0} H_{0}}\right) \cong y\left(\frac{c}{\left(R_{c}\right)_{t} H_{t}}\right) \\
& \frac{m_{p}}{m_{e}} \cong x y \cong 1838.8238 \\
& m_{e} c^{2} \cong x y^{\frac{1}{2}} \sqrt{\frac{e^{2} F_{X}}{4 \pi \varepsilon_{0}}} \cong x y^{\frac{1}{2}} \sqrt{\frac{e^{2} c^{4}}{4 \pi \varepsilon_{0} G_{A}}} \cong 0.5104 \mathrm{MeV}
\end{aligned}
$$

Now to a very good approximation muon and tau rest masses can be fitted as follows.

$$
\left(m_{\text {lepton }} c^{2}\right)_{n} \cong\left[\gamma^{3}+\left(n^{2} \gamma\right)^{n} \sqrt{N_{A}}\right]^{\frac{1}{3}} \sqrt{\frac{e^{2} c^{4}}{4 \pi \varepsilon_{0} G_{A}}}
$$

Where $\gamma \cong x y^{\frac{1}{2}} \cong x \sqrt{y} \cong 294.7074 y . n=1,2$. Please see the following table 2 [75]-[86],[90].

\begin{tabular}{ccc}
\multicolumn{3}{c}{ Table 2: To fit the muon and tau rest masses } \\
\hline No & $\begin{array}{c}\text { Obtained rest energy } \\
\text { of lepton in MeV }\end{array}$ & $\begin{array}{c}\text { Experimental rest energy } \\
\text { of lepton in MeV }\end{array}$ \\
\hline 1 & 105.908 & $105.6583668(38)$ \\
2 & 1775.97 & $1776.99(29)$ \\
3 & 42211.82 & Not detected \\
\hline
\end{tabular}

Proton rest energy can be expressed as follows. 


$$
\begin{aligned}
& m_{p} c^{2} \cong x^{2} y^{\frac{3}{2}} \sqrt{\frac{e^{2} F_{X}}{4 \pi \varepsilon_{0}}} \cong x^{2} y^{\frac{3}{2}} \sqrt{\frac{e^{2} c^{4}}{4 \pi \varepsilon_{0} G_{A}}} \cong 938.545 \mathrm{MeV} \\
& \left(m_{e} \text { and } m_{p}\right) c^{2} \cong(x)^{n}(y)^{(2 n-1) / 2} \sqrt{\frac{e^{2} F_{X}}{4 \pi \varepsilon_{0}}} \cong(x)^{n}(y)^{(2 n-1) / 2} \sqrt{\frac{e^{2} c^{4}}{4 \pi \varepsilon_{0} G_{A}}}
\end{aligned}
$$

Where $n=1$ and 2 . Neutron, proton and electron rest masses can be interrelated in the following way.

$$
\begin{aligned}
& \frac{m_{n} c^{2}-m_{p} c^{2}}{m_{e} c^{2}} \cong \ln (2 \sqrt{y}) \cong 2.524 \\
& m_{n} c^{2} \cong m_{p} c^{2}+\ln (2 \sqrt{y}) m_{e} c^{2} \cong 939.833 \mathrm{MeV}
\end{aligned}
$$

Within the nucleus, in a direct approach, proton-nucleon stability relation can be expressed as follows.

$$
\begin{aligned}
& A_{s} \cong 2 Z+\left(\frac{Z}{2 \sqrt{y}}\right)^{2} \cong 2 Z+\frac{Z^{2}}{4 y} \cong 2 Z+\frac{Z^{2}}{155.7} \\
& A_{s}-2 Z \cong \frac{Z^{2}}{155.7}
\end{aligned}
$$

Where $Z$ is the atomic number and $A_{s}$ is the corresponding stable mass number.

\section{Discussion}

Please note that even though it was having strong footing, Mach's principle [49], [50], [51] was not implemented successfully in modern physics and modern cosmology. One of the main motivations behind formulating the general theory of relativity was to provide a mathematical description to the Mach's principle. A very general statement of Mach's principle is "Local physical laws are determined by the large-scale structure of the universe". This concept was a guiding factor in Einstein's development of the general theory of relativity [93]. Einstein realized that the overall distribution of matter would determine the 'metric tensor', which tells the observer which frame is rotationally stationary. However, soon after its formulation, it was realized that the theory does not follow Mach's principle. As the theoretical predictions were matching with the observations, Einstein believed that the theory was correct and did not make any farther attempt to reformulate the theory to explain Mach's principle. Later on, several attempts were made by different researchers to formulate the theory of gravity based on Mach's principle. However most of these theories remain unsuccessful to explain different physical phenomena. The Einstein field equations are nonlinear and very difficult to solve. Einstein used approximation methods in working out initial predictions of the theory. But as early as 1916, the astrophysicist Karl Schwarzschild found the first non-trivial exact solution to the Einstein field equations, the so-called Schwarzschild metric [94], [41]. This solution laid the groundwork for the description of the final stages of gravitational collapse, and the objects known today as black holes. It can be suggested that, within the Hubble volume overall distribution of Hubble mass will explain the observed physical phenomena.

At any given cosmic time, the product of 'critical density' and 'Hubble volume' gives a characteristic cosmic mass and it can be called as the 'Hubble mass'. Interesting thing is that, Schwarzschild radius of the 'Hubble mass' again matches with the 'Hubble length'. Most of the cosmologists believe that this is merely a coincidence. At any given cosmic time, 'Hubble length' can be considered as the gravitational or electromagnetic interaction range. If one is willing to think in this direction, by increasing the number of applications of Hubble mass and Hubble volume in other areas of fundamental physics like quantum physics, nuclear physics, atomic physics and particle physics [7]-[14] slowly and gradually - in a progressive way, concepts of 'Black hole Cosmology' can be strengthened and can also be confirmed [36]-[41],[95]-[103]. The most interesting thing is that, at any given cosmic time, if the universe is a primordial growing black hole, then certainly its 'Schwarzschild radius' can be considered as its characteristic minimum size at that time. Clearly speaking, "forever rotating at light speed, high temperature and high angular velocity small sized primordial cosmic black hole gradually transforms into a low temperature and low angular velocity large sized massive primordial cosmic black hole". Based on the proposed relations and applications, Hubble volume or Hubble mass, can be considered as a key tool in unification as well as cosmology. Whether universe is a black hole or something else, one can find many interesting applications of Hubble volume and its corresponding Hubble mass in the current and past aspects of the universe. Hence magnitudes of Hubble length, Hubble volume and Hubble mass can be considered as characteristic back ground conditions for the observed atomic and cosmological physical phenomena.

Based on the proposed relations and concepts of black hole cosmology, definitions of cosmic homogeneity and cosmic isotropy must be re-addressed in a closed black hole universe. Independent of the redshift observations and with a great 
confidence now one can start seeing/observing the universe as a primordial expanding and light speed rotating black hole. From the above relations it is also clear that, now the black hole universe is expanding in a decelerating mode at a very small rate in such a way that with current technology one cannot measure its deceleration rate. Finally it can be suggested that cosmic acceleration and dark energy can be considered as pure mathematical concepts and there exists no physical base behind their confirmation. For the most serious cosmologists this may be bitter news, but it is a fact. Authors hope that, by 2015 definitely this subject will come into main stream physics

\section{Conclusion}

In a theoretical way, the proposed applications or semi empirical relations can be given a chance and the subject of elementary particle physics and cosmology can be studied in a unified manner. Now this is the time to revise the basics of 'quantum theory' of light. From cosmological time point of view the issue of 'constancy of quantum' (of angular momentum or energy) must be re-analyzed and resolved. By using the proposed relations and applying them in fundamental physics, in due course their role or existence can be verified. With these relations, Hubble constant can be estimated from atomic and nuclear physical constants. If one is able to derive them with a suitable mathematical model, independent of the cosmic redshift and CMBR observations, the future cosmic acceleration can be verified from atomic and nuclear physical constants. Now the key leftover things are nucleosynthesis and structure formation. The most important point to be noted here is that, synthesis of elementary physical constants seem to be more important and intrinsic than the 'cosmological nucleosynthesis'. Authors are working on this concept and will be discussed in detail in near future.

The study of cosmology, its progress and evolution is yet to be properly made to arrive at reliable and convincing conclusions. The present study is a major step forward in this direction. Even though there were a number of papers/books published on cosmology, the attempt for a comprehensive study on this subject, coupled with comparative studies with the modern cosmology on one hand and with the modern atomic physics on the other, was not made by anybody so far. Thus, the present study can be considered as a 'basic project' in this unified field. Cosmological observations through ground telescope or satellite telescope are a normal practice. In this paper under consideration, current cosmological changes can be understood by studying the atom and atomic nucleus through ground based experiments. It is an interesting part of the study of cosmology and fundamental interactions. This is quite unique and the openness in the subjects of cosmology and fundamental interactions can be eliminated. It is a challenging idea and 100 years of atomic, nuclear and cosmic physics can be refined and unified. Characteristic nuclear radius, rms radius of proton and the strength of electromagnetic interaction all seem to be the cosmological variables and observing the rate of change in their magnitude (on the cosmological time scale), the cosmic acceleration can be verified and thus the cosmic geometry can be confirmed from atomic, nuclear and particle physics. Without the advancement of nanotechnology or femto-technology this may not be possible. Independent of the cosmic red shift and CMBR observations cosmic acceleration can be checked in this new direction. With the proposed applications it is very clear to say that, without a combined and unified study of cosmology and atomic \& particle physics, one should not deny the concepts of black hole cosmology.

\section{Acknowledgements}

The first author is indebted to professor K. V. Krishna Murthy, Chairman, Institute of Scientific Research on Vedas (I-SERVE), Hyderabad, India and Shri K. V. R. S. Murthy, former scientist IICT (CSIR) Govt. of India, Director, Research and Development, I-SERVE, for their valuable guidance and great support in developing this subject. Same author is very much thankful to Dr. Sankar hazra and Dr. Timur Kamalov for their encouragement in presenting the subject in international conferences. Finally first author is thankful to Dr. Ramon A Isasi for his kind and valuable comments on this complicated subject.

\section{References}

[1] Peter Rowlands. Problems on the standard model of cosmology and the solutions arising from Santillie's theory. Hadronic Journal. Vol-35, No-6, 593-608, 2012.

[2] Mustapha Ishak, Remarks on the Formulation of the Cosmological Constant/Dark Energy Problems, Found Phys, 37,pp1470-1498, 2007.

[3] Bruno Leibundgut and Jesper Sollerman. A cosmological surprise: the universe accelerates. Europhysics News (2001) Vol. 32 No. 4

[4] P. J. E. Peebles and Bharat Ratra (2003). The cosmological constant and dark energy. Reviews of Modern Physics 75 (2): $559-606$

[5] Joshua Frieman, Michael Turner and Dragan Huterer, Dark Energy and the Accelerating Universe. Ann. Rev. Astron. Astrophys.46: pp385432, 2008.

[6] J. W. Moffat, Modified Gravity Or Dark Matter? Online Available: http://arxiv.org/abs/1 101.1935v2 
[7] Narlikar, J.V, Vishwakarma,R.G. and Burbidge.G., Interpretations of the Accelerating Universe, The Publications of the Astronomical Society of the Pacific, Volume 114, Issue 800, pp. 1092-1096, 2002.

[8] Saul Perlmutter, Supernovae, Dark Energy and the Accelerating Universe, American Institute of Physics, Physics Today, pp53-60, April 2003

[9] Arman Shafielooa, Varun Sahnib and Alexei A. Starobinsky, Is cosmic acceleration slowing down? Phys. Rev. D 80, pp101301, 2009.

[10] P. A. M. Dirac. The cosmological constants. Nature, 139, 323, 1937.

[11] P. A. M. Dirac. A new basis for cosmology. Proc. Roy. Soc. A 165, 199, 1938

[12] U. V. S. Seshavatharam, S. Lakshminarayana, Applications of Hubble Volume in Atomic Physics, Nuclear Physics, Particle Physics, Quantum Physics and Cosmic Physics. Journal of Nuclear Physics, Material Sciences, Radiation and Applications (JNPMSRA) Vol. 1, No. 1 August 2013 pp. $45-60$.

[13] Kragh, H. (1996). Cosmology and Controversy. Princeton University Press. p. 318. ISBN 0-691-02623-8.

[14] Hawking, S.W.; Ellis, G.F.R. (1973). The Large-Scale Structure of Space-Time. Cambridge University Press. ISBN 0-521-20016-4.

[15] Hawking S.W. A Brief History of Time. Bantam Dell Publishing Group. 1988

[16] Barbara Ryden. Introduction to Cosmology. The Ohio State University. January 13, 2006

[17] Christopher S. Reynolds. Astrophysics: Black holes in a spin. Nature. 494, 432-433 (28 February 2013)

[18] Michael J. Longo, Detection of a Dipole in the Handedness of Spiral Galaxies with Redshifts z 0.04, Phys. Lett. B 699, $224-229$ 2011.

[19] S.W. Hawking (1969). On the rotation of the universe. Mon. Not. Royal. Astr. Soc. 142, 129-141.

[20] C Sivaram and Kenath Arun, Primordial Rotation of the Universe, Hydrodynamics, Vortices and Angular Momenta of Celestial Objects. The Open Astronomy Journal, 2012, 5, 7-11

[21] S.-C. Su and M.-C. Chu. Is the universe rotating? Astrophysical Journal, 703 354.doi:10.1088/0004-637X/703/1/354

[22] Sidharth,B.G. Is the Universe Rotating? Prespacetime Journal. October 2010, Vol. 1, Issue 7, pp. 1168-1173.

[23] E. Kajari et al. Rotation in relativity and the propagation of light. Proceedings of the International School of Physics "Enrico Fermi", Course CLXVIII, pp. 45-148 (2009)

[24] Istvan Nemeti et al visualizing ideas about Godel-type rotating universes. Godel-type Spacetimes: History and New Developments.www.math-inst.hu/pub/algebraic-logic godunivisu-revised.pdf (2009)

[25] Marcelo Samuel Berman. A General Relativistic Rotating Evolutionary Universe. Astrophys. Space Sci.314:319-321,2008

[26] G. Chapline et al. Tommy Gold Revisited: Why Does Not The Universe Rotate? AIP Conf.Proc.822:160-165, 2006. http://arxiv.org/abs/astro$\mathrm{ph} / 0509230$.

[27] Robert V Gentry. New Cosmic Center Universe Model Matches Eight of Big Bang's Major Predictions without the F-L Paradigm. CERN preprint, EXT-2003-022, 14 Apr 2003

[28] Kurt Godel. Rotating Universes in General Relativity Theory. Proceedings of the international Congress of Mathematicians in Cambridge, 1: $175-81,1950$.

[29] Dmitri Rabounski. On the Speed of Rotation of Isotropic Space: Insight into the Redshift Problem. The Abraham Zelmanov Journal, Vol. 2, 2009, 208-223.

[30] Mauro Dorato. On becoming cosmic time and rotating universes. Time, Reality and Experience (provisional title), Royal Institute of Philosophy Series, Cambridge University Press, 2001.

[31] Yuri N. Obukhov. On physical foundations and observational effects of cosmic Rotation. Published in Colloquium on Cosmic Rotation, Eds M. Scherfner, T. Chrobok and M. Shefaat (Wissenschaft und Technik Verlag: Berlin, 2000) pp. 23-96. http://arxiv.org/abs/astroph/0008106v1,7August 2000.

[32] Barrow J D, Juszkiewicz R, Sonoda DH. Universal rotation - How large can it be? Mon Not R Astron Soc $1985 ; 213: 917$.

[33] U. V. S. Seshavatharam. Physics of rotating and expanding black hole universe. Progress in Physics. April, p 7-14, (2010).

[34] U.V.S. Seshavatharam. The Primordial Cosmic Black Hole and the Cosmic Axis of Evil. International Journal of Astronomy, 1(2): 20-37, (2012).

[35] U. V. S. Seshavatharam and S. Lakshminarayana. The reduced Planck's constant, Mach's principle, cosmic acceleration and the Black hole universe. Journal of Physical Science and Application 2 (10) (2012) 441-447.

[36] U. V. S. Seshavatharam, S. Lakshminarayana, B.V.S.T. Sai. Unified Concepts in Cosmic, Atomic and Nuclear Physics. Global Journal of Science Frontier Research ( A ) Volume XIII Issue I Version I Year 2012

[37] U. V. S. Seshavatharam, S. Lakshminarayana, Hubble Volume and the Fundamental Interactions, International Journal of Astronomy, Vol. 1 No. 5, 2012, pp. 87-100.

[38] U. V. S. Seshavatharam, S. Lakshminarayana, B.V.S.T. Sai. Is red shift an index of galactic'atomic light emission' mechanism? International Journal of Physics, Vol. 1, No.3, 49-64, (2013).

[39] U. V. S. Seshavatharam, S. Lakshminarayana, B.V.S.T. Sai. Nucleus, Atom and the Universe a combined study. International Journal of Advanced Astronomy, 1 (1), 1-12 (2013).

[40] Seshavatharam U.V. S. and Lakshminarayana. To confirm the existence of Black hole cosmology. International Journal of Advanced Astronomy, 2 (1), 21-36, 2013

[41] U. V. S. Seshavatharam. Light speed rotating black holes: The special holes. International Journal of Advanced Astronomy. 1 (1), 13-20 (2013).

[42] Hubble E. P, A relation between distance and radial velocity among extra-galactic nebulae, PNAS, 1929, vol. 15, 1929, pp.168-173.

[43] Hubble, E.P, The 200-inch telescope and some problems it may solve. PASP, 59, pp153-167, 1947.

[44] Alpher, R. A.; Herman, R. C. (1948). On the Relative Abundance of the Elements. Physical Review 74 (12): 1737-1742.

[45] Gamow, G. (1948). The Origin of Elements and the Separation of Galaxies. Physical Review 74 (4): $505-506$

[46] Gamow, G. (1948). The evolution of the universe. Nature 162 (4122): 680-682

[47] Dicke, R. H. (1946). The Measurement of Thermal Radiation at Microwave Frequencies. Review of Scientific Instruments 17 (7): $268-275$

[48] Sciama, D. W. (1971). Modern Cosmology. Cambridge: Cambridge University Press. OCLC 6931707.

[49] Narlikar J.V. Direct Particle Formulation of Mach's Principle. Einstein Studies, vol. 6: Mach's Principle: From Newton's Bucket to Quantum Gravity, pp. 250-261, 1995 Birkhauser Boston, Inc. Printed in the United States

[50] Bondi, Hermann; and Samuel, Joseph (July 4, 1996). "The Lense-Thirring Effect and Mach's Principle". arXiv:gr-qc/9607009 [gr-qc]. doi:10.1016/S0375-9601(97)00117-5

[51] Marmet. A New Non-Doppler Redshift. Physics Essays, 1(1):24-32, 1988. Online

[52] Louis Marmet. On the Interpretation of Red-Shifts: A Quantitative Comparison of Red-Shift Mechanisms. www.marmet.org/louis/index.html

[53] Seshavatharam U.V. S. and Lakshminarayana S. Peculiar Relations in Cosmology. Volume 2 Progress in Physics, April, (2013).

[54] U. V. S. Seshavatharam and S. Lakshminarayana. Is Planck's constant a cosmological variable? International Journal of Astron-omy, 2(1): 1115 DOI: 10.5923/j.astronomy. 20130201.02 (2013).

[55] Recami E. Elementary Particles as Micro-Universes, and "Strong Black-holes": A Bi-Scale Approach to Gravitational and Strong Interactions. Preprint NSF-ITP-02-94. Posted in the arXives as the e-print physics/0505149, and references therein. 
[56] Geiger H and Marsden E. On a diffuse reaction of the particles. Proc. Roy. Soc., Ser. A 82: 495-500, 1909.

[57] H. Yukawa. On the Interaction of Elementary Particles. Proc. Phys. Math. Soc. Jap. 17 (48). 1935

[58] P. J. Mohr and B.N. Taylor, CODATA Recommended Values of the Fundamental Physical Constants.2007. http://physics.nist.gov/constants

[59] Michael O. Distler et al. The RMS Charge Radius of the Proton and Zemach Moments. Phys. Lett.B. 696: 343-347,2011

[60] C. L. Bennett et al, Nine-Year Wilkinson Microwave Anisotropy Probe (WMAP) Observations: Final Maps and Results. Submitted to Astrophysical Journal Supplement Series. http://arxiv.org/abs/1212.5225v1.

[61] J. Huchara. Estimates of the Hubble Constant, 2010. Harvard-Smithsonian Center for Astrophysics. http://hubble.plot.dat

[62] W. L. Freedman et al. Final Results from the Hubble Space Telescope Key Project to Measure the Hubble Constant. The Astrophysical Journal 553 (1): 47-72. 2001.

[63] J. Beringer et al. Particle Data Group. Phys. Rev. D86, 010001 (2012)

[64] J.K. Webb et al. Indications of a spatial variation of the fine structure constant. Physical Review letters, 107 (19) 2011

[65] Jon O' Bryan et al. Constraints on Spatial Variations in the Fine-Structure constant from Planck. http://arxiv.org/pdf/1306.1232v1.pdf

[66] J.V. Narlikar, Introduction to cosmology, Cambridge Univ. Press, 2002, 393- 400.

[67] Craig J. Copi et al. Big bang nucleosynthesis and the baryon density of the universe. Science 267, 192-199, 1995. http://arxiv.org/abs/astroph/9407006v2.

[68] Max Planck. On the Law of Distribution of Energy in the Normal Spectrum. Annalen der Physik, vol. 4, p. $553 \mathrm{ff}$ (1901).

[69] Satyendranath Bose. Planck's law and the light of quantum hypothesis. American Journal of Physics, Vol.44 No.11, November 1976. J. Astrophys. Astr. (1994) 15, 3-7

[70] Shashikant Gupta. Balckbody radiation. JAP 2003, Indian Institute of Science. 5 Dec 2003.

[71] Jonathan M. Marr and Francis P. Wilkin. A better presentation of Planck's radiation law. ArXiv: 1109.3822v3 [astro-ph.SR] 13 Mar 2012. Accepted to the American Journal of Physics.

[72] Lianxi Ma et al. Two forms of Wien's displacement law. Lat. Am. J. Phys. Educ. Vol. 3, No. 3, Sept. 2009

[73] Jensen, William B. (July 2003). The Universal Gas Constant R . J. Chem. Educ. 80 (7): 731.

[74] Peter Denenhower. Is there a connection between Planck's constant, Boltzmann's constant and the speed of light? Thesis - Simon Fraser University, 1987.

[75] Curtis J. Forsy. The Resonance structure of matter, nature of gravitation, and the quantum energy states of the Hydrogen atom. Physics Essays: June 2009, Vol. 22, No. 2, pp. 112-121.

[76] U. V. S. Seshavatharam and S. Lakshminarayana. Strong Nuclear Gravitational Constant and the Origin of Nuclear Planck Scale. Progress in Physics, Vol 3, pp. 31-38, July, 2010

[77] U. V. S. Seshavatharam and S. Lakshminarayana, Role of Avogadro number in grand unification. Hadronic Journal. Vol-33, No 5, 2010 Oct. p513.

[78] U. V. S. Seshavatharam and S. Lakshminarayana, To confirm the existence of atomic gravitational constant. Hadronic journal, Vol-34, No 4, 2011 Aug. p379.

[79] U. V. S. Seshavatharam and S. Lakshminarayana.. SUSY and strong nuclear gravity in (120-160) GeV mass range. Hadronic journal, Vol-34, No 3, 2011 June, p.277

[80] U. V. S. Seshavatharam and S. Lakshminarayana.. Strong nuclear gravity - a brief report. Hadronic journal, Vol-34, No 4, 2011 Aug.p.431.

[81] U. V. S. Seshavatharam and S. Lakshminarayana. Nucleus in Strong nuclear gravity. Proceedings of the DAE Symp. on Nucl. Phys. 56 (2011) p.302.

[82] U. V. S. Seshavatharam and S. Lakshminarayana. Atom, universe and the fundamental interactions. Global Journal of Science Frontier Research (A) Vol. 12 Issue 5, p.1, (2012).

[83] U. V. S. Seshavatharam and S. Lakshminarayana.. Accelerating universe and the expanding atom. Hadronic journal, Vol-35, No 3, 2012 p. 271 .

[84] U. V. S. Seshavatharam and S. Lakshminarayana. Past, present and future of the Avogadro number. Global Journal of Science Frontier Research (A) Vol. 12 Issue 7, p.27, (2012).

[85] U. V. S. Seshavatharam and S. Lakshminarayana. To understand the four cosmological interactions. International Journal of Astronomy 2012, 1(5): $105-113$

[86] U. V. S. Seshavatharam and S. Lakshminarayana. Molar electron mass and the basics of TOE. Journal of Nuclear and Particle Physics 2012, 2(6): $132-141$

[87] U. V. S. Seshavatharam and S. Lakshminarayana. Logic Behind the Squared Avogadro Number and SUSY International Journal of Applied and Natural Sciences. Vol. 2, Issue 2, May 2013, 23-40

[88] U. V. S. Seshavatharam and S. Lakshminarayana, Super Symmetry in Strong and weak interactions. Int. J. Mod. Phys. E, 19(2): 263, 2010.

[89] U. V. S. Seshavatharam and S. Lakshminarayana. Integral charge SUSY in Strong nuclear gravity. Proceedings of the DAE Symp. On Nucl. Phys. 56: 842, 2011.

[90] U. V. S. Seshavatharam and S. Lakshminarayana. Role of SUSY in finding the equivalent term for the first three terms of the semi empirical mass formula. To be published in Proceedings of the DAE Symp. On Nucl. Phys. 2013, Mumbai, Inda.

[91] J. Beringer et al. Particle Data Group. Phys. Rev. D86, 0100012012.

[92] Abdus Salam. Einstein's Last Dream: The Space -Time Unification of Fundamental Forces, Physics News, $12(2): 36$, June 1981.

[93] David Gross, Einstein and the search for Unification. Current science, Vol. 89, No. 12, 25 Dec 2005.

[94] Einstein A. Die Grundlage der allgemeinen Relativit atstheorie, Annalen der Physik, 1916, v. 354, 769-822

[95] Schwarzschild K. On the gravitational field of a point mass according to Einstein's theory. Abraham Zelmanov Journal, 2008, vol. 1, 10-19

[96] Pathria, R. K. The Universe as a Black Hole. Nature 240 (5379):298-299.doi:10.1038/240298a0 (1972).

[97] Good, I. J. Chinese universes. Physics Today 25 (7): 15. July. doi:10.1063/1.3070923 (1972).

[98] Poplawski, N. J. Radial motion into an Einstein-Rosen bridge. Physics Letters B 687 (23): 110113. (2010).

[99] Zhang, Tianxi. A New Cosmological Model: Black Hole Universe. Progress in Physics, 3: 3-11, (2009).

[100] Zhang, Tianxi. Cosmic microwave background radiation of black hole universe. Astrophysics and Space Science, Volume 330, Issue 1, pp 157-165. (2010).

[101] Joel Smoller and Blake Temple. Shock-wave cosmology inside a black hole. Proc Natl Acad Sci U S A. September 30; $100(20)$ : 1121611218. (2003).

[102] Andy Gardner, Joseph P. Conlon. Cosmological natural selection and the purpose of the universe. Complexity Volume 18, Issue 5, pages 4856, May/June 2013

[103] Chul-Moon Yoo et al. Black Hole Universe: Time evolution. arXiv:1306.1389v1 [gr-qc] 6 Jun (2013). 